\title{
Artikel
}

Lars Koch*

\section{Über artivistische Interventionen. Invektivität, Medien, Moral}


Abstract: On the basis of two aesthetic interventions of Christoph Schlingensief and the Center for Political Beauty (ZPS) this article analyses the relationship between artivism and invectivity. In each case the underlying theatrical dispositifs are being discussed. With this, the article inquires into the respective procedures of disturbing the audience and the public. Whereas Schlingensief's politics of form aims at creating a sphere of ambiguity, the ZPS is all about stimulating moral indignation. This also reflects the diametrically opposed capabilities of artivistic art: Controversy on the one hand, partisanship on the other.

Keywords: Invektivität, Artivismus, konfliktuelle Ästhetik, Schlingensief, ZPS, Rancière - Invectivity, Artivism, conflictual aesthetics, Schlingensief, ZPS, Rancière

*Prof. Dr. Lars Koch, Technische Universität Dresden, Institut für Germanistik, Professur für Medienwissenschaft und Neuere deutsche Literatur, lars.koch@tu-dresden.de

Das Verletzen und Beleidigen, die Beschimpfung des Publikums und auch die Missachtung von Regeln des guten Geschmacks gehören zum bewährten Affizierungsinstrumentarium moderner Aktionskunst. Spätestens seit den künstlerischen Avantgarden des frühen 20. Jahrhunderts ist die Transgression moralischer oder ästhetischer Konventionen eine weit verbreitete Modalität der Aufmerksamkeitserzeugung, mit der die kunsträsonierende Öffentlichkeit immer zu rechnen hat. Filippo Marinettis ästhetische Feier der Gewalt (Manifest des Futurismus, 1909) oder auch André Bretons Forderung, mit einem Revolver in die Menge zu schießen (Zweites Manifest des Surrealismus, 1930), sind nur zwei Beispiele für diese Selbstinszenierung des Künstlers als Souverän, der der entstehenden Massengesellschaft ebenso verachtend gegenüber zu stehen behauptet wie dem Normenrepertoire einer bürgerlichen Kunst- und Kulturgesinnung.

Von den avantgardistischen Anfängen der Verbindung von Authentizitätsinszenierung, Gewaltpose und Invektiv-Kommunikation lässt sich über Fluxus in der Musik oder Martin Kippenberger und die anderen ,neuen Wilden' der späten 1970er und 1980er Jahre in der Malerei eine Verbindungs- linie ziehen zu aktuellen Avantgarde-Protokollen der Skandalisierung und des Resonanzkalküls, wie sie etwa für Santiago Sierra, Jonathan Meese oder Mathieu Malouf charakteristisch sind. In den hier zur Schau gestellten Inszenierungen - man denke etwa an Sierras Aktion, in der er arbeitslosen kubanischen Jugendlichen einen Strich auf den Rücken tätowierte ${ }^{1}$ oder an Maloufs antisemitische Skulptur \#luketurnerisretarded ${ }^{2}$ - eines invektiven Zynismus, setzt sich eine Strategie des selbstreferenziellen Trollings in Szene, die manchmal dem ironischen Nihilismus der Alt Right nahe kommt. ${ }^{3}$ Angetrieben von der Affordanz der sozialen Medien, die eine "digitale Affektkultur

$1250 \mathrm{~cm}$ Line Tattooed on 6 Paid People Espacio Aglutinador, Havana, Cuba, December 1999, vgl. https:// sammlung.staedelmuseum.de/de/werk/250-cm-line-tattooed-on-6-paid-people-espacio-aglutinador (letzter Zugriff 20.04.2020).

2 Vgl. Stromberg (2019) Los Angeles gallery opens show. 3 "Dadurch, dass die Ironie in der Lage ist, ethische Fragen zu durchkreuzen, wird die Umkehrung der ihr anhängigen sozialen Funktionen ermöglicht, sodass sie im Aufgeben des Politischen die Herrschaft nicht mehr hinterfragt, sondern affirmiert. Ironie bestätigt hier diejenige Ideologie, die sie abzuwerten oder zu ignorieren vorgibt, indem sie jede potenzielle oder vermeintliche Alternative negiert." 
des Extremen"4 begünstigt, realisiert sich hier ein aufmerksamkeitsökonomisch informiertes Beleidigungsprogramm, das einmal mehr die ästhetische Provokation zum zentralen Mittel der Steigerung von Popularität macht. Invektivität dient dabei als Ressource einer affizierungsintensiven Selbstreferenz, die das Soziale und das Politische den eigenen, kunstinternen Codes unterwirft.

Einer anderen funktionalen Logik - so scheint es zumindest - folgt die Strategie invektiver Adressierung von Publikum und Öffentlichkeit im Kontext aktueller ästhetischer Interventionen, die eine direkte soziale und politische Wirksamkeit der Kunst realisieren wollen. Auch im Kontext dieser theatralen "Gesellschaftsspiele"5 kommen Techniken der konfrontativen DissensProduktion zum Einsatz, nur dient die Erregung der Öffentlichkeit in diesem Fall einem anderen Ziel. Wie schon das Label Artivismus vermuten lässt, geht es in der programmatischen Annäherung von Kunst und sozialer Bewegung hier darum, die Gesellschaft mit den Mitteln der Kunst mindestens zu irritieren und im besten Falle auch zu verändern. Während Künstler wie Mathieu Malouf, Jonathan Meese oder auch Damien Hirst die Transgression als Möglichkeit radikaler KunstAutonomie zelebrieren, betonen Vertreter*innen des Artivismus das Primat eines politisch-ethischen Projektes, dessen kommunikativer Ausgestaltung die künstlerische Darstellung zu dienen habe. Invektivität wird hier zur vielgenutzten Ressource eines ästhetischen Engagements, das sich im Sinne autonomer Heteronomie mehr oder weniger ästhetisch vermittelt in den Dienst einer politischen Sache stellt. Sie findet „als herabsetzende Identifizierung von Personen oder Gruppen"6 Verwendung mit dem Ziel, die Zuschauenden zu Positionierung herauszufordern und sie so aus der ihnen zugeschriebenen konsumistischen Indifferenz herauszuholen.

In Aneignung der ursprünglich politisch-militärischen Semantik ${ }^{7}$ geht es bei der ästhetischen

Pinto/Stakemeier (2019) Glossar, S. 95. Vgl. hierzu auch Colebrook (2000) Meaning of irony, v.a. S. 7.

4 Reckwitz (2017) Gesellschaft der Singularitäten, S. 270. 5 Vgl. Malzacher (2020) Gesellschaftsspiele.

6 Häusler/Heyne/Koch/Prokic (2020) Verletzen und Beleidigen, S. 309.

7 Verb franz. ,intervenir', lat. ,interventio', ,einschreiten, vermitteln', im 17. Jahrhundert aus dem Französischen entliehen, ab dem 19. Jahrhundert vor allem in der Bedeutung
Intervention meist um eine temporäre Aktion im öffentlichen Raum, die mit dem Ziel verbunden ist, die öffentliche Wahrnehmung zu schärfen, zu provozieren und mit Blick auf kulturelle, soziale und politische Fragen und Themen zu entautomatisieren. ${ }^{8}$ Happening und Flashmob als Paradebeispiele für interventionistische Kunst etwa leben von einer performativen Praxis, die sowohl für Performer*innen als auch für Zuschauende eine situativ außeralltägliche und mit hohem Irritationspotenzial angereicherte Wirklichkeit schafft. ${ }^{9}$ Beruhend auf einer Strategie des Eingreifens in oder des Unterbrechens von öffentlicher Ordnung leisten ästhetische Interventionen insofern eine spezifische Arbeit am symbolischen Repertoire der Gesellschaft: Sie greifen kulturelle Routinen an, geben ihnen so "den in der fortlaufenden Pragmatik ihrer Anwendung vergessenen Charakter radikaler Fragwürdigkeit"10 zurück und machen damit latente Alternativen sicht- und erfahrbar. ${ }^{11}$ Schon bei Guy Debord und den Situationist*innen ging es im Sinne Antonio Gramscis um eine Störung des common sense, ${ }^{12}$ um eine gegenhegemoniale "Arbeit, die von Individuen und Kollektiven ohne Legitimation geleistet wird, die versuchen, die Karte des Möglichen neu zu zeichnen."13 Ästhetische Interventionen, die es sich als "Dynamisierung der soziopolitischen Ordnung" 14 zur Aufgabe machen, den gesellschaftlichen Konsens aufzustören, wollen den kollektiven Möglichkeitssinn stimulieren, indem sie die unhinterfragte Selbstverständlichkeit der gesellschaftlichen Verhältnisse unter Stress setzen. Diese Verfremdung der sozialen Wirklichkeit geschieht in der Hoffnung, in den ästhetisch organisierten Erfahrungen von

, sich in die Angelegenheiten eines anderen Staates einmischen'. Im Kontext des Militärischen: RAF: Gewaltsame Aktionen als ,revolutionäre Interventionen' der politischen Avantgarde; politisch-militärische Interventionen als Streitfall des Völkerrechts, insofern damit der in der UN-Charta von 1949 verankerte ,Grundsatz souveräner Gleichheit' und damit auch das Prinzip der Nichteinmischung verletzt wird.

8 Zum Konzept der Entautomatisierung vgl. den klassischen Text von Šklovskij (1969) Kunst als Verfahren. 9 Vgl. Warstat et al. (2015) Theater als Intervention.

10 Lehmann (2011) Wie politisch ist das postdramatische Theater?, S. 35.

$11 \mathrm{Vgl}$. zum kritischen Potenzial des Prinzips ästhetischer Störungen Koch/Nanz/Pause (2018) Disruptions in the Arts. 12 Vgl. Snir (2016) Not just one common sense.

13 Rancière (2011) Moments politiques, S. 14.

14 Surmann (2014) Ästhetische In(ter)ventionen, S. 23. 
Ambiguität den herrschenden Eindruck von Alternativlosigkeit als Produkt hegemonialer Anstrengungen durchsichtig und problematisch werden zu lassen. ${ }^{15}$

Artivistische Interventionen können dort, wo das ästhetische Kalkül aufgeht und die taktisch eingesetzte Invektive ein Feuerwerk von erbosten Anschlusskommunikationen zündet, dazu beitragen, "die von der Erfindung der Gleichheit verursachten Risse im Herrschaftsgefüge spürbar zu machen und die Denkoperationen, die sie andauernd zu kitten versuchen, aufzudecken." ${ }^{16}$ Artivistische Invektiv-Kommunikation wirkt dann, wenn sie mit einer Ästhetik der prekären Exponierung operiert, wie ein Brandbeschleuniger, der situativ die sozialen Positionen der beteiligten Akteur*innen und Zuschauer*innen entsichert, eine öffentliche Situation erschütternder Desorientierung erzeugt und damit politische Zuschreibungspraktiken zur Disposition stellt. In diesem Sinne realisiert das theatrale Dispositiv des Artivismus performativ ein "Exponierungsgeschehen $[\ldots]$, in dem sich das Ausgesetztsein an den anderen und damit die Potenzialität der Verletzung, Beleidigung, Herabsetzung als eine Grundbedingung von Subjekt und Sozialität ausdrückt. So verweist die theatrale Exponiertheit auf eine genuine Verletzbarkeit des Subjekts, auf seine Angst vor der Verletzung, in der jede Machtbeziehung begründet ist. "17 Die politische Dimension ästhetischer Interventionen manifestiert sich so in einem "Konflikt über das Dasein einer gemeinsamen Bühne, über das Dasein und die Eigenschaften derer, die auf ihr gegenwärtig sind." ${ }^{18}$ Wo dies passiert, ist die Invektive nicht mehr bloßes Mittel zum Zweck der Aufmerksamkeits- und Affektmobilisierung, sondern artikuliert zugleich auch - als implizites Insis-

15 Dass diese Interventionsmuster in den letzten Jahren unter Anleitung von Götz Kubitscheck von der Identitären Bewegung (IB) angeeignet wurden, verweist auf eine derzeit zu konstatierende Krise der emanzipativ orientierten künstlerischen Kritik. Bemerkenswert ist zudem, dass nicht alleine die kommunikativen Strategien, sondern auch die Formen und ästhetischen Verfahren der Aufstörung adaptiert wurden. Vgl. hierzu die Analyse von Ulrich (2017b) Schönheit; Wagner (2017) Angstmacher, S. 128-153; Koch (2020) Gefühlspolitik, S. $102 f$.

16 Rancière (2011) Moments politiques, S. 12.

17 Häusler/Heyne/Koch/Prokic (2020) Verletzen und Beleidigen, S. 308.

18 Rancière (2002) Unvernehmen, S. 38. Vgl. hierzu auch Wihsturz (2014) Streit um die Bühne. tieren oder explizit ausformuliert - ein Nachdenken über den gesellschaftlichen und symbolischen Ermöglichungszusammenhang von Invektiv-Kommunikation. Dergestalt auf die Ebene von Metainvektivität ${ }^{19}$ transponiert, diskutiert die ästhetische Intervention das spannungsgeladene Verhältnis von "Subjektsein, Sprechen und Vernommenwerden"20 und bezieht auch die eigene Position im sozialen Geschehen wechselseitiger invektiver Zuschreibungen, des Aneinander-Vorbeiredens und der (produktiven) Missverständnisse mit ein. Entscheidend für eine solche interventionistische Politik der Form ist dabei, die manifeste Konstellation von Macht, Ausbeutung, Diskriminierung und Herabsetzung nicht alleine als Ausdruck konkreter politischer Programme und Ideologien zu kritisieren, sondern sie zugleich in einem prinzipiellen Sinne als Effekt einer ganz basalen „Aufteilung des Sinnlichen"21 zu fassen, die als hegemoniale Habitualisierung von Seh- und Wahrnehmungsgewohnheiten den kollektiven Vorstellungshaushalt der Gesellschaft formatiert.

Eine andere Strategie, die gerade unter den Bedingungen der aktuellen gesellschaftlichen "Diskursverwilderung"22 auf viel Resonanz in Öffentlichkeit und sozialen Medien stößt, besteht in der willentlichen Forcierung von dichotomen Konfliktlagen. Während die zuvor kurz skizzierte Politik der Form auf eine Verunordnung der bestehenden Ordnungen - der Politik, des Sozialen, des Diskurses oder auch des Sinnlichen - hinarbeitet, geht es in der alternativen Variante um das Programm einer Zuspitzung: Letztere nutzt Invektivität vornehmlich als Ressource, mit deren Hilfe politische Konfliktlagen - zumindest rhetorisch in eine Logik des Antagonismus übersetzt werden sollen. Im Rahmen eines solchen Wirkungskalküls intensivierter Konfrontation trägt Invektiv-Kommunikation im Regime des Ästhetischen dann dazu bei, Subjektpositionen zu verhärten, positiv wie negativ konnotierte Identitätszuschreibungen zu stabilisieren und die Konfliktualität des Sozialen aus dem Register des Politischen in jenes der Moral zu übertragen. Während Invektivität im ers-

$19 \mathrm{Vgl}$. zum Konzept des Metainvektiven die Überlegungen von Scharloth (2017) Hassrede und Invektivität.

20 Kleesattel (2016) Politische Kunst-Kritik, S. 41.

21 Rancière (2006) Aufteilung des Sinnlichen.

22 Pörksen/Schulz von Thun (2020) Kunst des Miteinander-Redens, S. 10. 
ten Fall als produktiver Faktor zu einem politischen Widerstreit beiträgt, ist sie im zweiten Fall bloßes Mobilisierungsvehikel einer selbstsicheren moralischen Positionierung, von der aus der unübersichtliche, komplexe Wirkungszusammenhang der Politik anhand eindeutig gezogener Demarkationslinien überblickt und gedeutet werden kann.

Wenn vor dem Hintergrund dieser allgemeinen Überlegungen zu Invektivität als Thema und Verfahren des Artivismus im Folgenden mit Christoph Schlingensiefs Containeraktion Bitte liebt Österreich von 2001 und der Aktion Flüchtlinge Fressen des Zentrums für Politische Schönheit (ZPS) von 2016 zwei Beispiele ästhetischer Intervention diskutiert werden sollen, dann geht es dabei vor allem um zwei Dinge: Zum einen ist genauer zu rekonstruieren, wie die invektive Aufstörung von Publikum und Öffentlichkeit als Instrument und Effekt der jeweiligen Aktion funktioniert. Es soll also an Schlingensief und dem ZPS exemplarisch die invektive Imprägnierung des Konzeptes artivistischer Intervention vorgeführt werden. ${ }^{23}$ Zum anderen soll daran anknüpfend die generelle Beobachtung diskutiert werden, dass die Ausprägung und Nutzung invektiv-gesättigter kommunikativer Muster zum Kernbestand im ästhetischen Repertoire aktionistischer Kunstformen im Dienste der Re-Artikulation politischer Positionen gehört. Invektive Formen sind Elemente jener interventionistischer Resonanzkalküle, mittels derer sich im Kampf um gesellschaftliche Sprechund Handlungslizenzen ein An- und Übergriff auf

23 Dass dabei nicht im literarisch-poetologischen Sinne von einer ,invektiven Gattung' zu sprechen ist, liegt auf der Hand, besteht das ,Gattungsmerkmal' des Artivismus - gerade im ästhetischen Regime der Kunst - doch in der situativen Hybridisierung unterschiedlicher Kunstformen, Medien und Formate. Ästhetische Interventionen schöpfen ihr disruptives Potenzial gerade aus der Negation von Ordnung und Erwartbarkeit. Dies gilt für ihre Fremdreferenz (soziale Konventionen, Normalität etc.) wie für ihre Selbstreferenz (Gattungsregeln, ästhetische Klassifikationen etc.). Genau diese programmatische Verweigerung von Regelhaftigkeit wird in Teilen von Publikum und Öffentlichkeit als Provokation und Herabsetzung eigener Wertvorstellungen erlebt. Allerdings birgt diese Affizierungsstrategie eine aufmerksamkeitsökonomische Überbietungslogik, die sich nicht bis ins Unendliche steigern lässt. Darin, dass auch die artivistische Bewirtschaftung des Invektiven in ein Spiel von Erwartungserwartungen eingebunden ist und sich mithin abnutzen kann, liegt ein weiterer Grund für die aktuelle Krise der künstlerischen Kritik. die routinierten Prozeduren der hegemonialen Herstellung von Normalität bewerkstelligen lässt.

Während damit zunächst das Element invektiver Adressierung als wiederkehrendes Moment artivistischer Interventionen herausgestellt wird, sollen im Anschluss mit der Gegenüberstellung von Schlingensief und dem ZPS zugleich auch zwei diametral entgegengesetzte Konzepte der politischen Wirksamkeit von Aktionskunst kontrastiert werden. Während bei Schlingensief die Produktion ästhetischer Widersprüchlichkeit zum Einsatzpunkt von politischem Widerspruch wird, geht es beim ZPS-Vordenker Philipp Ruch und seinen Mitstreiter*innen um das Vor-Augen-Stellen einer konkreten Handlungsalternative zur dominierenden politischen Praxis, deren durch die Aktion herausgestellte invektive Evidenz die politisch Mächtigen unter Legitimations- und Handlungsdruck setzen soll.

\section{Schlingensiefs Bilderstörung: Der Container als Invektiv-Arena}

Schlingensiefs Aktionskunst der 1990er und frühen 2000er Jahre arbeitet in unterschiedlichen ästhetischen Konstellationen mit der invektiven Infragestellung des common sense. Allen voran die Aktion Bitte liebt Österreich, die im Jahr 2000 anlässlich der Wiener Festwochen und vor dem Hintergrund der Überprüfung der Einhaltung der europäischen Grundrechte durch die EU stattfand, realisiert unterschiedliche, aber miteinander interferierende Konstellationen des Invektiven. So steht im Zentrum der Aktion die Produktion und Zirkulation liminaler Bilder, deren Invektivierungspotenzial Schlingensief nutzt, um den Rassismus von Massenmedien und Politik ${ }^{24}$ zum Gegenstand einer intensiven öffentlichen Debatte zu machen. Schlingensief selbst hat diese Container-Aktion als "Bilder-Störungsmaschine" bezeichnet. ${ }^{25}$ Mit dem Programm der invektiven Bilderstörung interveniert er in einen populistischen Diskurs, der identitätspolitisch entlang einer Unterscheidung des Eigenen und

24 Vor allem der Kronen Zeitung und der Freiheitlichen Partei Österreichs (FPÖ).

25 Schlingensief (2014) Ich weiß, S. 105. 
des Fremden operiert und dabei affektpolitisch auf die provokative Thematisierung von Ressentiment und Diskriminierung setzt. Die Aktion spielt die Konsequenzen einer rigiden, normalerweise aber im medialen Off vollzogenen Abschiebepraxis auf spektakuläre Weise durch. Ihre elementaren Bestandteile und Ereignissequenzen rücken in den Fokus, was sonst nicht zum Thema wird, weil es nicht zum Selbstbild der freundlichen, offenen Gesellschaft passt: der Container auf einem zentralen Platz der Wiener Innenstadt, die mehrtägige Dauer der Aktion inklusive allabendlicher Abschiebe-Performance von jeweils zwei Asylbewerber*innen, die textuelle Rahmung durch die am Container angebrachten Spruchbänder ("Ausländer raus" und "Unsere Ehre heißt Treue") ${ }^{26}$, die fremdenfeindlichen Wahlplakate der FPÖ samt Parteifahne sowie die Entgrenzungen in Massenmedien und Internet, schließlich die Einmischung von FPÖ-Verantwortungsträgern, Anzeigen von Privatleuten und entsprechende Ermittlungen der Staatsanwaltschaft Wien wegen Verunglimpfung des Staates. Das derart ausgestaltete Geschehen zielt im invektiven Vor-Augen-Stellen auf eine Situation der Positionierungsnotwendigkeit $a b$, in der niemand mehr neutrales Publikum sein kann, alle dafür aber affektiv und politisch beteiligte Akteur*innen werden. Die Container-Aktion führt dementsprechend vor, dass man sich angesichts des Wiedererstarkens rassistischer und xenophober Inhalte in der politischen Kultur nicht nicht positionieren kann. Damit ist Bitte liebt Österreich ein gutes Beispiel für eine konfliktuelle Ästhetik, wie sie Oliver Marchart als Praxis der "Antagonisierung" 27 beschreibt:an aesthetics that is conflictual in a double sense: it conflicts with the aesthetics of the spontaneous ideologists of the art field (the aesthetics of simplistic complexity), and it seeks to work out the political implications of conflictual artistic practices. It is, in this sense, both a conflicting aesthetics and an aesthetics of conflict. ${ }^{28}$ Schlingensiefs Container-Aktion funkti-

26 Diesen SS-Spruch hatte kurz zuvor der niederösterreichische FPÖ-Parteiobmann beim Landesparteitag seinem Publikum zugerufen, vgl. o. A. (2000) Aufregung.

27 Marchart (2000) Licht des Antagonismus, S. 254.

28 Marchart (2019) Being agitated, S. 23. Marchart geht von einer strikten Dichotomie zwischen zwei Positionen aus, von denen die eine die politische Dimension der Kunst in einer Arbeit an der Aufteilung des Sinnlichen betont (Ästhetik simpler Komplexität) und die andere, von ihm oniert in diesem Sinne als Kunst radikal politisch, weil sie direkt in eine konkrete Diskurskonstellation interveniert, darüber hinaus aber aufgrund des ihr zugrundeliegenden ästhetischen Dispositivs eine Form der Öffentlichkeit erzeugt, in der Identitäts- und Zuschreibungskonflikte in ihrer ganzen dissensuellen Wucht artikuliert werden können. ${ }^{29}$ Angesichts der Vielzahl der zum Teil unfreiwillig Beteiligten - Passant*innen, Medienvertreter*innen, Performer*innen usw. - und der mehrtägigen Dauer der Aktion ist es nicht mehr Schlingensief alleine, der mit der Unterbrechung kommunikativer Routinen die Aktion durchführt. Vielmehr entwickelt sich, angestoßen durch die invektive Bildpolitik der Container-Aktion, eine politische Eskalationsdynamik, die ihrerseits die medialen Regulationsroutinen unterbricht. Im Fortgang der Aktion kommt es in tumultartigen Szenen zu einer Überblendung unterschiedlicher politischer Positionen, die sich wechselseitig in ihrer vermeintlichen Deutungssicherheit irritieren und einander in Widersprüche verwickeln. Wenn gegen Ende der Aktion, linke' Protestierende den Container stürmen, um die Asylbewerber*innen symbolisch zu befreien, ist damit zugleich eine wohl kaum intendierte Reparaturarbeit am öffentlichen Bild Österreichs verbunden, insofern sie destruktiv auf jene Installation einwirken, über die sich gerade FPÖ, Kronen Zeitung und andere konservative und rechte Kräfte empören. Indem so die wohltemperierten Unterscheidungskriterien der Ortung und Ordnung politischer Gegner*innenschaft unterminiert werden, entstehen die Bedingungen der Möglichkeit einer Situation des Streitens, aus der eine neue politische Debatte erwachsen kann.

Um ihr ganzes Potenzial der Aufstörung entfalten zu können, müssen Schlingensiefs Aktionen ein Maximum an affektiver Beteiligung erreichen. $\mathrm{Zu}$ den entsprechenden Affizierungsstrategien, derer sich seine artivistischen Aktionen - allen voran der Wiener Container - bedienen, gehören

bevorzugte, in einem manifesten Sinne über „Agitation, Propaganda und Organisation" zu einer politischen Stellungnahme und Positionierung drängt. Vgl. ebd. S. 14-18 und S. 30-35. Die hier eingenommene Perspektive auf Schlingensief schlägt demgegenüber vor, Bilderstörung als Verbindung von konkreter politischer Positionierung und Politik der Form als zwei Ebenen eines übergeordneten kritischen Projekts zu lesen.

29 Vgl. Rancière (2000) Konsens, Dissens, Gewalt. 
Techniken der Verkörperung und der Visualisierung, die abstrakte Diskurspositionen in affektgeladene Bilder und Handlungssequenzen übersetzen. Dadurch, dass Schlingensief im Internet über die Abschiebung der einzelnen ,internierten' Asylsuchenden abstimmen lässt, macht er die praktischen Konsequenzen der nationalkonservativen Politik der FPÖ durch eine Individualisierung der Betroffenen sichtbar. Die Aktion, die nicht nur an das in Deutschland erstmalig im Jahr 2000 ausgestrahlte, populäre "Eliminationsspiel" ${ }^{30} \mathrm{Big}$ Brother anknüpft, sondern zugleich das Lager als bio-politisches Paradigma der Moderne aufruft, ${ }^{31}$ kreiert in inrer Anlage als Aufführung von Exklusion eine kontroverse Auseinandersetzung um Redepositionen, Solidarisierungsansprüche und Vereinnahmungen. Deren Sogwirkung betrifft Politiker*innen der FPÖ ebenso wie die Journalist*innen der Kronen Zeitung und anderer Medien, Passant*innen, Internet-User*innen, politische Aktivist*innen und Schlingensief selbst. Der konzeptionelle Faden der Aktion besteht darin, einen interpassiven, ironisch-distanzierten Konsum der Performance nach besten Kräften zu verunmöglichen. ${ }^{32}$ Bitte liebt Österreich hat auf einer Ebene erster Ordnung einen Appellcharakter. Die Aktion will Positionierung und Parteinahme motivieren, wenn nicht erzwingen. Hier geht es Schlingensief darum, politische Bemühungen der suggestiven Affekt- und Aufmerksamkeitslenkung bis zur Kenntlichkeit zu entstellen. Schon das Happening des Container-Einzugs vom 9. Juni, das mit Scheinwerfern und Blaskapelle seinen eigenen Ereignischarakter betont, irritiert dadurch, dass die Formen des Entertainments zwar aufgerufen, aber nicht in ein stimmiges Gesamtbild überführt werden. Mittels der Strategie einer vorsätzlichen Überaffirmation wird es möglich, die mediale Logik der populistischen FPÖ-Politik von innen heraus

30 So Sloterdijk (2001) Gespräch.

$31 \mathrm{Vgl}$. den überpointierten Essay von Agamben (2002) Homo Sacer.

32 Robert Pfaller hat als kritisches Korrelat zur Interaktivität das Konzept der Interpassivität vorgeschlagen: Im Sinne einer Kunstrezeption, in der das Kunstwerk die Rezipierenden in die Lage versetzt, ihr ,Genießen' von vornherein an das Kunstwerk zurück zu delegieren, versteht Pfaller Interpassivität als Form einer risikolosen Beteiligung, d.h. "als Abwesenheit von Interaktivität”, als „negative Größe" der Interaktivität. Pfaller (2008) Ästhetik der Interpassivität, S. 103. in die eigene Aporie zu treiben. Im Zentrum der Bildstörung steht dabei der Container als Installation der Absonderung, die als ein dystopischer Nicht-Ort mitten in Wien als Störkörper platziert, die Grenzen zwischen innen und außen, Peripherie und Zentrum, Befehl und Gehorsam, Verfügungsmacht und Verfügungsmasse zum affektiv aufgeladenen, anderen Bild werden lässt, das die Bilder- und Image-Politik der Regierung Schüssel in einer Kaskade von performativen "Operationen, die eine Abweichung hervorrufen"33, als politische Inszenierung durchsichtig werden lässt. Störung funktioniert hierbei als Wahrnehmungsverschiebung. Während Bundeskanzler Wolfgang Schüssel darum bemüht war, die Politik seiner rechts-konservativen Regierungskoalition in einen Rahmen von Normalität zu stellen und die politische Debatte damit von einer Ebene der Fundamentalkritik weg auf eine der Detailkritik zu lenken, kehrt die Container-Aktion die Invisibilisierung der problematischen Ausgangsvoraussetzungen veränderter normativer Standards um und rückt sie wieder ins Zentrum der Aufmerksamkeit. Aus einem looking through wird ein looking at, das hinterfragt, welche politischen Werte schon als gesetzt gelten, bevor das politische Alltagsgeschäft einsetzt.

Dementsprechend geht es Schlingensief nicht alleine um den Transport konkreter politischer Inhalte. Im Gegenteil dient sein provokatives Spiel mit Zitaten und Phrasen vor allem dazu, Widerspruch zu erzeugen und eine gesellschaftliche Debatte zu initiieren. Das Wirkungskalkül der Aktion geht daher auf einer Ebene zweiter Ordnung davon aus, dass die invektivitätsgetriebene affektive Beteiligung und die Vielstimmigkeit und Unübersichtlichkeit der Adressierungen eine Situation der politischen Emergenz entstehen lassen, in deren konfliktueller Komplexität die Kontingenz aller politischen und sozialen Ordnungen offensichtlich wird.

Wie Jacques Rancière feststellt, ist die gegenwärtige ,Postdemokratie' von einem neoliberalen Realismus bestimmt, der demokratische Meinungsbildung suggeriert, eigentlich aber Alternativlosigkeit konstatiert und so Utopien diskreditiert. ${ }^{34}$ Gegen eine solche Stillstellung des Möglichkeitssinns setzt Rancière - und die Aktionskunst Chris-

33 Rancière (2005a) Bestimmung der Bilder, S. 14. 34 Vgl. Rancière (2002) Unvernehmen, S. 105-120. 
toph Schlingensiefs folgt ihm darin - die Denkfigur des ,Unvernehmens'. Gemeint ist ein prinzipieller Dissens, der den "Anteil der Anteillosen"35 am politischen Prozess ins Blickfeld rückt und damit die impliziten kategorialen und normativen Voraussetzungen für Gleichheit, Partizipation und Empowerment ins Zentrum der Aufmerksamkeit gerückt sieht: Das Unvernehmen ist nicht der Konflikt zwischen dem der weiß und jenem, der schwarz sagt. Es ist der Konflikt zwischen dem der ,weiß' sagt und jenem der auch ,weiß' sagt, aber der keineswegs dasselbe darunter versteht bzw. nicht versteht, dass der andere dasselbe unter dem Namen der Weiße sagt. [...] Das Unvernehmen betrifft weniger die Argumentation als das Argumentierbare, die An- oder Abwesenheit eines gemeinsamen Gegenstands zwischen einem $X$ und einem Y. Es betrifft das sinnliche Darstellen dieses Gemeinsamen [...]. ${ }^{36}$ Schlingensiefs Intervention setzt genau hier ein: Indem er die im politischen Diskurs sonst bloß als stumme Kennzahl anwesenden Asylbewerber*innen in die Kunstproduktion einbezieht und ihr prekäres Dasein in die menschenunwürdige Konstellation des Containers überträgt, realisiert er ein Staging der von der österreichischen Dominanzkultur weitestgehend invisibilisierten Praxis der rassistisch konnotierten Diskriminierung. ${ }^{37}$

In der Summe fungiert die Container-Aktion als Positionierungen erzwingender Angriff auf das die öffentliche Debatte dominierende österreichische Selbstverständnis. Schlingensiefs Arrangements aus "Schlagbildern"38, Performance-Ele-

35 Rancière (2008) Zehn Thesen zur Politik, S. 11.

36 Rancière (2002) Unvernehmen, S. 9f.

$37 \mathrm{Zu}$ Schlingensiefs Arbeit am Sichtbarkeitsregime der Gesellschaft vgl. auch Häusler/Heyne/Koch/Prokic (2020) Verletzen und Beleidigen, S. 193-258.

38 Allen voran das Container-Banner "Ausländer raus", dem als medial zirkulierendem Schlagbild mit Michael Diers gesprochen "sowohl eine prägnante Form als auch ein gesteigerter Gefühlswert eigentümlich ist, insofern [es ...] entweder einen bestimmten Standpunkt für oder wider ein Streben, eine Einrichtung, ein Geschehnis nachdrücklich beton[t] oder doch wenigstens gewisse Untertöne des Scherzes, der Satire, des Hohnes und dergleichen deutlich mit erklingen [lässt]. Dem Schlagwort, das nicht selten eine Zeit oder Zeitströmung auf einen stimmigen, mitunter auch polemischen Begriff zu bringen vermag und in aller Munde ist, antwortet mit dem Schlagbild in ähnlicher Funktion eine ubiquitäre, ganz auf Wirkung verlegte, eindrückliche Darstellung, seien es z.B. Spott-, Reklame- oder Pressebilder." Diers (1997) Schlagbilder, S. 7. menten und Diskursartikulationen produzieren eine Ereignis- und Äquivalenzkette im öffentlichen Raum, die aufgrund ihrer Provokationskraft kaum ignoriert werden kann. Mehr noch: Durch die im Dispositiv der Aktion angelegten Feedbackschleifen, die immer neue Kaskaden von Anschlusskommunikationen herausfordern, wächst sich die Intervention zu einer Wunde in der Repräsentations- und Redeordnung aus, die eine Eskalationsspirale in Gang setzt, an deren Ende offen darüber gestritten wird, wer im öffentlichen Raum reden darf und wer nicht, wem es zuzuhören gilt und wo die Grenzen des Sagbaren verlaufen. Angesichts des Containers ist es - zumindest für die Dauer der Aktion - nicht mehr möglich, das konstitutive Zusammenspiel von nationaler Identitätspolitik, gewaltsamer Reinheitsfantasie und rassistischer Xenophobie, das in der Politik der Regierung Schüssel politische Macht übernommen hat, in die Latenz der Gesellschaft zu verdrängen.

Die Container-Aktion ist vor allem eine Auseinandersetzung mit der affektiven Macht einer massenmedial orchestrierten "Politik der Bilder"39, die in der Festlegung von Sichtbarkeitsregimes die gesellschaftlich formierte Vorstellung von Normalität konfigurieren. Insofern damit zugleich auch Interpellationsweisen und Subjektivierungsprozesse zum Thema werden, fungiert Bitte liebt Österreich als eine Erschütterung selbstverständlicher und als solche immer schon intuitiv vorausgesetzter und situativ aktualisierter Selbst- und Fremdbilder. Gerade weil die Atmosphäre auf dem Wiener Theaterplatz sich in der Konfrontation unterschiedlicher Meinungen von Tag zu Tag mehr erhitzt, entsteht ein unübersichtlicher Möglichkeitsraum, in dem politische Positionen, Konflikte und Koalitionen, die sonst in der Latenz der privaten Alltagskommunikation verbleiben, deutlich hervortreten. Im Spektrum an öffentlichen Verhaltensweisen, das von verbaler Beleidigung und Krawall bis an die Grenze zur körperlichen Auseinandersetzung reicht, geraten die Dinge, Festlegungen und Zuschreibungen in Bewegung und werden zur Verhandlungsmasse. Schlingensiefs Bilderstörungsmaschine realisiert damit eine subversive Gegenstrategie, die ein Spiel von Positionswechseln und Funktions-

39 Vgl. Rancière (2005a) Bestimmung der Bilder. 
änderungen in Gang setzt. ${ }^{40}$ Es geht dabei nicht alleine darum, den Rechtspopulismus der FPÖ zu kritisieren. Vielmehr schafft die Container-Aktion ein kulturelles und politisches Forum, in dem ein Widerstreit darüber, was Öffentlichkeit eigentlich bedeutet, neu entstehen kann. In diesem Sinne markiert Schlingensiefs Statement, "Widerstand ist vorbei, Sie müssen Widersprüchlichkeit erzeugen"41, gleich mehrere Fluchtlinien der Aktion: Zunächst geht es um die Identifikation und Affektion der Zuschauer*innen mit einem Geschehen der Selbst- und Fremdpositionierung, in einem zweiten Schritt soll genau diese konstitutive Positionalität selbst reflektiert werden. Wenn dies gelingt, wenn aus der Erfahrung der Widersprüchlichkeit unmittelbar oder vermittelt durch die die Aktion begleitenden Paratexte ein Willen zum Widerspruch wird, der immer auch ein "wieder miteinander sprechen" jener bedeutet, die im Streit ein "politisches Band"42 erschaffen haben, dann verwandelt sich die Invektiv-Arena des Wiener Theaterplatzes in eine Agora, auf der ein produktiver Widerstreit möglich werden kann. ${ }^{43}$ In der Konsequenz scheint dann eine Dimension der Kritik als Form von Entunterwerfung auf, wie sie von Judith Butler im Abschluss an Michel Foucault als Fluchtlinie eines politischen Prozesses der Emanzipation skizziert wurde:

Man könnte auch sagen, das Subjekt ist gezwungen, sich in Praktiken zu formen, die mehr oder weniger schon da sind. Vollzieht sich diese Selbst-Bildung jedoch im Ungehorsam gegenüber den Prinzipien, von

40 Vgl. hierzu Koch (2014) Performance als Bilderstörungsmaschine.

41 Siehe das Interview mit Christoph Schlingensief im Begleitmaterial zur DVD Ausländer Raus! Schlingensiefs Container, Bonus Film-GmbH, (Ö 2000), Hoanzl Ö 2002.

42 Rancière (2002) Unvernehmen, S. 148.

43 "Arenen sind [...] Handlungs- und Kommunikationsräume, die mit bestimmten Sprech- und Handlungslizenzen, Konventionen sowie Erwartungshaltungen beziehungsweise Aufmerksamkeitsmustern einhergehen. [...] Sie bilden häufig manifest oder latent eine gesellschaftliche Ordnung ab. Sie sind der Ort antagonistischer, agonistischer, theatraler, tribunaler Aushandlungen von identitätsbildenden Deutungen sozialer Ordnungen. Invektiven brauchen Arenen als Ermöglichungszusammenhang, gleichzeitig haben Invektiven die transformatorische Kraft, Arenen instabil werden zu lassen, zu verschieben oder überhaupt erst zu erzeugen, insofern beispielsweise durch eine Invektive ein Publikum generiert wird." Häusler/Heyne/Koch/Prokic (2020) Verletzen und Beleidigen, S. 306f. denen man geformt ist, wird Tugend jene Praxis, durch welche das Selbst sich in der Entunterwerfung bildet, was bedeutet, dass es seine Deformation als Subjekt riskiert und jene ontologisch unsichere Position einnimmt, die von neuem die Frage aufwirft: Wer wird hier Subjekt sein, und was wird als Leben zählen, ein Moment des ethischen Fragens, welcher erfordert, dass wir mit den Gewohnheiten des Urteilens zu Gunsten einer riskanteren Praxis brechen, die versucht, den Zwängen eine künstlerische Leistung abzuringen ${ }^{44}$

\section{Ruchs moralische Anklage: Der Käfig als Tribunal}

Das zweite hier zu diskutierende Beispiel - das Zentrum für politische Schönheit (ZPS) - stellt in Deutschland derzeit die wohl aufmerksamkeitsökonomisch erfolgreichste Agentur im Dienste ästhetischen Engagements für politische Veränderung dar. Für Philipp Ruch, die zentrale Figur des ZPS, bedeutet insbesondere das katastrophale Geschehen an der Außengrenze Europas und im Mittelmeer ein "Interventionsgebot", das seine von einem "aggressive[n] Humanismus" 45 angeleitete Aktionskunst auf einen direkten Wirkungsanspruch und das Streben nach sozialer Unmittelbarkeit verpflichtet. Kunst, so ist es in der Selbstbeschreibung des ZPS zu lesen, müsse "reizen, wehtun und verstören"46, um damit Einfluss auf Politik und öffentliche Debatte nehmen zu können. Um das „große Unrecht" 47 zu beheben, dessen sich Europa angesichts der Not der geflüchteten Menschen schuldig mache, bedürfe es drastischer Mittel, die dazu in der Lage seien, die deutsche Bevölkerung moralisch zu mobilisieren und die politischen Entscheidungsträger*innen entsprechend unter Druck zu setzen. Kunst, wie das ZPS sie versteht, tendiert damit zur Kunstmission, sie fungiert als "rather straight, in your-face-propaganda" 48 , die eindeutig politische Position bezieht und unmissverständliche Botschaften formuliert.

44 Butler (2009) Was ist Kritik?, S. 249.

45 Vgl. Ruch (2013) Aggressiver Humanismus.

46 So die programmatische Selbsterklärung auf der Homepage des ZPS, https://politicalbeauty.de/ (letzter Zugriff 27.04.2020).

47 So eine Beschreibung der Ausgangslage auf der Internet-Seite zur Aktion Flüchtlinge fressen, https://politicalbeauty.de/ff.html (letzter Zugriff 27.04.2020).

48 Marchart (2019) Being agitated, S. 21. 
Seit seinen ersten Aktionen, den Lethe-Bomben von 2009 und der Säule der Schande von 2010 agiert das ZPS mit der Zielstellung der Konsensstörung im Register des Invektiven. Dabei geht es dem ZPS darum, der Gesellschaft disruptiv einen Spiegel vorzuhalten und sie durch aufsehenerregende Aktionen auf ihre blinden Flecken im Umgang mit den Konsequenzen der deutschen und europäischen Außen- und Wirtschaftspolitik hinzuweisen. Generell sind die artivistischen Interventionen des ZPS vor allem darauf angelegt, eine symbolische Wiederkehr der verdrängten Gewalt der außereuropäischen Peripherien im Zentrum der deutschen Dominanzkultur zu erreichen und daraus kommunikativ einen Impuls zu einem Politikwechsel abzuleiten. Unter dieser Stoßrichtung kristallisieren sich im Aktionsspektrum des ZPS mehrere Themenkonjunkturen heraus. Während es in den letzten Jahren vor allem um eine kritische Auseinandersetzung mit der deutschen Flüchtlingspolitik ging, rückt nunmehr, sekundiert von Ruchs Manifest Schluss mit der Geduld (2019) das Verhältnis der deutschen Demokratie zur Alternative für Deutschland (AfD) und zum Rechtsextremismus ins Zentrum der künstlerischen Beschäftigung.

Die inszenatorischen Mittel, die Ruch und seine Mitstreiter*innen zur Intervention in die deutsche Diskurslandschaft nutzen, sind um Drastik und Deutlichkeit der Aussage bemüht. Das ZPS rekurriert immer wieder auf das grundgesetzlich verankerte Recht der Kunstfreiheit, um invektive Grenzüberschreitungen zu bewerkstelligen. So schrieb etwa die Aktion 25.000 Euro Belohnung (2012) die Eigentümerfamilie der Rüstungsfirma Krauss-Maffei Wegmann symbolisch zur Fahndung aus, was u.a. zu einer Unterlassungsklage und heftigen Debatten im Feuilleton führte. Die späteren Aktionen Erster europäischer Mauerfall (2014), Die Toten kommen (2015) und Flüchtlinge fressen - Not und Spiele (2016), die sich alle drei mit der von den Massenmedien organisierten Verdrängungsleistung der Gesellschaft auseinandersetzen, arbeiten mit Mitteln der Spektakularisierung von Not, Leid und Sterben, um den prekären Status quo der deutschen und europäischen Flüchtlingspolitik in Frage zu stellen. Vor allem die Aktion Flüchtlinge fressen, für die das ZPS am 16. Juni 2016 pünktlich zur Fußball-Europameisterschaft eine römisch anmutende Arena mit vier Tigern in der Mitte Berlins eröffnete, überschreitet konsequent die Grenze zwischen Kunstaktion und politischem Engagement. Dabei ging es konkret darum, durch die Erzeugung moralischer Empörung eine Änderung der gesetzlichen EinreiseRegelungen für geflüchtete Menschen zu erzwingen. Konzeptionell als ticking-bomb-scenario angelegt, forderte das ZPS Geflüchtete dazu auf, "sich im Widerstand gegen Deutschlands tödlichstes Gesetz"49 fressen zu lassen. Von dem so in ein Tribunal verwandelten Tigerkäfig geht die alarmierende Suggestion eines Handlungsnotstands aus, der - situiert in einem irritierenden Graubereich zwischen Fakten und Fiktionen - Politik und Öffentlichkeit auf eine virtuelle Anklagebank zerrt und zur Rechtfertigung zwingt. ${ }^{50}$ Da die zeitliche Struktur der Aktion als ein sich über mehrere Tage hinwegziehender Countdown organisiert ist, an dessen Schluss das Opfer steht, entsteht eine Dringlichkeit, der sich niemand ohne moralische Kollateralschäden entziehen kann. Dies betrifft die verantwortlichen Politiker*innen, die durch die Aktion direkt adressiert und in den Fokus der medialen Beobachtung gestellt werden, ebenso wie das Publikum, das vor Ort wie auch in den sozialen Medien affektiv angesprochen und in eine gemeinsame Erfahrung der moralischen Parteinahme eingebunden werden soll.

Genau in dieser Produktion von Parteilichkeit und Anteilnahme, die nicht nur moralische Entrüstung, sondern zugleich auch einen fundamentalen Zweifel an den Entscheidungsroutinen der repräsentativen Demokratie insinuiert, setzt die Affektpolitik von Flüchtlinge fressen an. Die brachial gestaltete Aktion, die „ein Denkmal für unsere Zeit" 51 sein will und ganz bewusst mit der schon von Niklas Luhmann beschriebenen Relevanzordnung der Massenmedien spielt, ${ }^{52}$ nimmt

49 Gemeint war der $\S 63$ des Aufenthaltsgesetzes, der festlegt, dass Flüchtlinge nicht per Flugzeug nach Deutschland kommen dürfen.

50 Damit knüpft die Aktion direkt an das berühmte Zitat „Die Scene wird zum Tribunal" aus Schillers Ballade Die Kraniche von Ibykus (1797) an, der damit auf die Verwandlung des Publikums in einen Gerichtshof anspielte. Heute nutzt vor allem Milo Rau das Theater als Ort der Verhandlung von gesellschaftlichen Konflikten, etwa in seiner Aktion Das Kongo-Tribunal von 2015.

51 So die Selbstbeschreibung im Internet-Auftritt der Aktion, https://politicalbeauty.de/ff.html (letzter Zugriff 27.04.2020).

52 Vgl. Luhmann (1996) Realität der Massenmedien, Kapitel 5. 
die im Framing der Gladiatorenspiele ausgestellte Spektakelorientierung der medialen Aufmerksamkeitsökonomie auch im Arrangement der Installation wieder auf:

Die gladiatorische Interpretation Europas: Die Arena ist der Bautypus einer verdichteten tödlichen Falle. Hier werden vor großem Publikum Schicksale generiert - Niederlagen und Siege. Spektakel machen den ideologischen Kern einer Gesellschaft, etwa Flüchtlinge im Namen der Sicherung der EU-Außengrenzen sterben zu lassen, sichtbar. In den Pausen der Abschreckungspolitik wird die Arena gesäubert und frischer Sand gestreut. ${ }^{53}$

Interessant ist das Dispositiv der Aktion zudem, weil es die welterzeugenden Effekte des medialen Sichtbarkeitsregimes in den Fokus nimmt. In der gestalteten Struktur des öffentlichen Raumes, der sich analog zu Schlingensiefs Container - als räumliche Realisierung einer zugrundeliegenden Aufteilung des Sinnlichen verstehen lässt, wird deutlich, dass Wahrnehmung immer zugleich Filterung und Priorisierung bedeutet: Während bestimmten Zonen des Politischen, Sozialen und Kulturellen kollektive Bedeutung zugeschrieben wird, werden parallel hierzu aufgrund medialer Rasterungen bestimmte Komplexe des Leidens und der Verantwortlichkeit unsichtbar und fallen aus dem Spektrum möglicher diskursiver Bezugnahmen heraus. Wie schon bei der Aktion Die Toten kommen begegnet auch Flüchtlinge fressen der massenmedialen Übersetzung existenziellen Leids in statistische Kurven und abstrakte Kennzahlen mit einer Körperpolitik, die scheinheiligen bzw. ignoranten Wahrnehmungsroutinen eine Form körperlicher Präsenz - der sterblichen Überreste, des kommenden Opfers - entgegenstellt. Die durch die Aktionen realisierte Präsenz von realen Körpern stört als nicht auszublendende Materialität das medial vermittelte Selbstbild einer offenen Gesellschaft, indem es dessen Abhängigkeit von einer permanenten Normalisierungsleistung aufzeigt, die eine dichotome Logik von innen und außen und eine implizite politische Essentialisierung der Migrant*innen als radikal Andere zur Voraussetzung hat.

Diese Mechanik der Invisibilisierung übersetzt sich vor dem Berliner Gorki-Theater in eine Architektur der Gleichzeitigkeit des Ungleichzeitigen: So

53 Internet-Auftritt der Aktion, https://politicalbeauty.de/ ff.html (letzter Zugriff 27.04.2020). war neben der Frontscheibe des gläsernen Käfigs eine große Leinwand angebracht, die im Verlauf der Aktion, akustisch begleitet von einem Meeresrauschen, das symbolisch an das Massengrab Mittelmeer erinnert, auch Spiele der Fußball-Europameisterschaft übertrug. Diese Gegenüberstellung, die die Frage nach einer kollektiven Begehrensstruktur, seinen nationalen Objekten und seinen invektiven Implikationen aufwirft, verleiht dem Untertitel der Aktion "Not und Spiele" eine Konnotation, die die täglichen Ansagen der TigerDompteure und die begleitenden Diskussionen mit Publizist*innen, Wissenschaftler*innen und Politiker*innen in die grundsätzliche Perspektive sozialer Mobilisierungsfähigkeit rückt. Angesichts der großen Affekt-Ereignisse nationaler Selbstberauschung, wie sie z.B. eine Fußballeuropameisterschaft darstellt, spielt der Überlebenskampf an den Peripherien der westlichen Welt - wenn überhaupt - nur eine untergeordnete Rolle. Dieser gerinnt in den allabendlichen Bildern überfüllter Flüchtlingsboote zu einer medialen Szenographie der gefährlichen Welt, wie sie da draußen eben ist. Das Leid der Anderen, so die bittere Quintessenz, tritt nur dann in das Aufmerksamkeitsfenster der medialen Berichterstattung, wenn es sich in das narrative Muster des Melodramas einpassen lässt, wenn es also individualisierbar, adressierbar, situierbar erscheint. Und selbst dann gerinnt es nur zu kleinen, kurzfristigen Emotionsclustern, aus denen nichts folgt, was über den Moment intensiver Erregung hinausginge. Paradigmatisch für das Anforderungsprofil massenmedialer Affektbewirtschaftung steht etwa das Foto des kleinen Alan Kurdi, dessen Leichnam im September 2015 an der türkischen Mittelmeerküste angeschwemmt wurde. Dem deutschen Pressekodex zum Trotz druckte die Bild-Zeitung das Konterfei des 3-jährigen toten Kindes ab - semantisch gerahmt von Mitleids- und Empörungsbekundungen und diffusen Forderungen, dem Sterben im Mittelmeer ein Ende zu bereiten. Diese Form der zynischen Instrumentalisierung, die tiefenstrukturell auf eine Konsumtion des Spektakulären abzielt, bildet einen der kritischen Bezugspunkte der Aktion Flüchtlinge fressen, die damit implizit Judith Butlers Frage nach graduellen Unterschieden der Betrauerbarkeit des Todes in die Performance hineinholt. ${ }^{54}$ Dass diese

54 Vgl. zu Judith Butlers Begriff der ,Grievability' Butler (2015) Precariousness and grievability. 
Meta-Dimension ${ }^{55}$, die die Aktion im Hinblick auf die Bedingungen der Möglichkeit ästhetischer Intervention in Zeiten gegenwärtiger Aufmerksamkeitsökonomien hin öffnet und an die Medien zurückspielt, seitens des Feuilletons wie auch von der Politik weitestgehend ausgeblendet wurde, kann wenig überraschen. Über die rund zweiwöchige Dauer von Flüchtlinge fressen hinweg stand dort vor allem der Vorwurf der Geschmacklosigkeit und des Zynismus im Vordergrund der Debatte. Die diskursive Rahmung, die das ZPS im Kontext des Begleitprogramms sicherzustellen versuchte, wurde quasi von einer Welle der öffentlichen Aufgeregtheit hinweggespült. Exemplarisch für den massiven Handlungsdruck, den das ZPS mit seiner Aktion auf politische Entscheidungsträger*innen auszuüben versuchte, aber auch für deren erfolgreiche Umlenkungsstrategie, die darauf abzielte, das Objekt der moralischen Entrüstung auszutauschen, stehen etwa zwei Tweets des Bundesministeriums des Inneren (BMI) vom Juni 2016, die die politischen Fragen der Aktion ignorieren und stattdessen auf bürgerlichen Anstand und guten Geschmack abheben.

Dass die ,Krisen'-Kommunikation zwischen vermeintlich unpersönlichen Institutionen (BMI vs. ZPS) hier über soziale Netzwerke erfolgt, ist indes Teil eines von der Aktion inszenierten Spiels, das gerade dann im Sinne der Aktion erfolgreich gespielt wird, wenn es seinen Spielcharakter aufs Spiel setzt und in Richtung politischer Realität entgrenzt. In dem Moment, in dem das BMI in

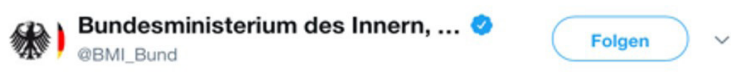

Unser Kommentar zu \#fluechtlingefressen:

Aktion ist zynisch \& wird auf dem Rücken der Schutzbedürftigen ausgetragen.

Abb. 1: Beweislastumkehr.

Tweet des Bundesministeriums des Innern, für Bau und Heimat (@BMI_Bund) am 17.06.2016; https://twitter.com/ BMI_Bund/status/743794217651150849

55 Bezeichnend für die ästhetische Strategie des ZPS ist es, dass auf der Homepage der Aktion ein ähnliches Bild zu finden ist. Die abgebildete Frauenleiche am Strand hat die Funktion, die Aktion mit maximalem moralischen Druck anzureichern. Wie schon bei Die Toten kommen übernimmt der Geflüchtetenkörper dabei die Funktion eines Affekt-Vehikels. Vgl. hierzu Koch/Prokic (2022) Weißen der Kritik. den Dialog einsteigt, vermischen sich zwei kommunikative Codes mit zunächst zu unterscheidenden Wirklichkeitsbezügen. Die vom ZPS entworfene und durch Video-Clips veranschaulichte Fiktion einer anders organisierten Migrationspolitik in die Bundesrepublik Deutschland erfährt durch die Reaktion des Ministeriums einen neuen Status als faktischer Debattenbeitrag. Der damit verbundene diskurspolitische Zugewinn wird auch nicht dadurch gemindert, dass die Tweets des BMI durchaus als herablassende Belehrung über ein zivilgesellschaftliches Fehlverhalten gelesen werden konnten. Denn gerade der moralische Vorwurf des Zynismus, der sich in der Klage der Kunstkritik doppelt, und die Klage darüber, dass die Aktionen des ZPS kaum von einem Medienevent zu unterscheiden seien, ruft eine grundsätzliche Frage der kulturräsonierenden Öffentlichkeit

\section{Vernunft und geltendes Recht behalten Oberhand: Flug ist storniert}

Die Initiatoren der Aktion "Flüchtlinge fressen" ("Zentrum für politische Schönheit") und der Fake-Website

www.sanktionen-bmi.de hatten angekündigt, am heutigen Tag rund 100 Flüchtlinge illegal aus der Türkei nach Berlin einzufliegen. Für den Flug waren "Spenden" gesammelt worden. Die Fluggesellschaft hat heute Morgen mitgeteilt, dass der Flug storniert wurde.

Zudem war angekündigt worden: Sofern bis heute nicht § 63 Abs. 3 des Aufenthaltsgesetzes abgeschafft sei, würden Flüchtlinge getötet, indem sie Tigern "zum Fraß vorgeworfen" werden. Angeblich hätten sich Flüchtlinge "freiwillig" hierfür gemeldet. Die genannten Tiere wurden in den letzten Tagen in Berlin in einem Käfig öffentlich ausgestellt.

Politik und Regierung zu kritisieren, ist erlaubt und wichtig. Nicht zuletzt deshalb stellt sich das BMI Diskussionen zu seinen Themen bewusst, gerne und täglich - im Social Web und an vielen anderen Stellen.

Hier handelt es sich jedoch um eine geschmacklose Inszenierung, bei der die betreffenden Flüchtlinge von den Initiatoren instrumentalisiert werden.

Deutschland hat seit Beginn des Syrien-Konflikts rund 400.000 syrische Flüchtlinge aufgenommen und nimmt - insbesondere im Rahmen des geordneten 1:1-Resettlements - weiter Syrer aus der Türkei auf.

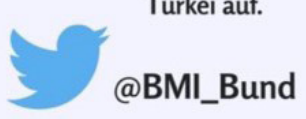

Abb. 2: Geschmacksurteil als Immunisierung

Tweet des Bundesministeriums des Innern, für Bau und Heimat (@BMI_Bund) am 28.06.2016; https://twitter. com/BMI_Bund/status/747707681247473665 
auf, die auch bei Schlingensiefs Aktionen immer im Raum stand: Ist das Kunst oder kann das weg?

An der Persistenz, mit der sich das ZPS kontinuierlich dem Vorwurf der Eventisierung ausgesetzt sieht, lässt sich eine hegemoniale Normalisierungsstrategie nachvollziehen: Das Störpotenzial artivistischer Interventionen soll dadurch reduziert werden, dass deren Kunststatus - und damit ihre Lizenz zu provozierender Drastik und invektiver Deutlichkeit - in Frage gestellt wird. Mit dem Versuch einer Skandalisierung, die zugleich eine rhetorische Diskreditierung der Inhalte der jeweiligen Interventionen durchzusetzen versucht, sah sich auch der "Politikclown"56 Christoph Schlingensief immer wieder konfrontiert. Aufgeregte, aggressive und herablassende Anschlusskommunika-tionen sind im Rahmen der ästhetischen Dispositive artivistischer Interventionen, die ja Invektivität als Ressource affektiver Aufstörung nutzen, eine logische Folge der Mediengesellschaft. Erst die Feedbackschleifen zwischen Performance, Medien, Politik und Öffentlichkeit initiieren jene Eskalationsdynamik, aus der die Aktionen ihre Durchschlagskraft zu gewinnen hoffen. Dass die Erregtheit der Debatte allerdings noch kein hinreichendes Indiz für die Radikalität der Intervention und die mit ihr verbundenen Potenziale einer nachhaltigen Politisierung ist, wird deutlich, wenn man abschlieBend noch einmal im Vergleich der Aktionen Bitte liebt Österreich und Flüchtlinge fressen genauer auf die jeweiligen Publikumskonzepte und deren Aktivierungslogiken schaut. Auch wenn das ZPS immer wieder darum bemüht ist, sich in eine Kontinuität zur Aktionskunst Schlingensiefs zu stellen, fallen doch mehr Unterschiede als Gemeinsamkeiten auf. Wo Schlingensief darauf aus war, im Dissens eine Möglichkeit der (Re-)Politisierung zu schaffen, geht es dem ZPS vor allem darum, Zustimmung für die eigene Position zu formieren.

\section{Invektivität als Ressource und Problem artivistischer Intervention}

Mit Blick auf das Thema dieses Sonderhefts erscheint ein Vergleich der Aktionen Bitte liebt Österreich und

56 Gilcher-Holtey (2019) Skandalisierung des Skandals.
Flüchtlinge fressen über thematische Kontinuitäten hinaus vor allem aus zwei Gründen interessant. Zum einen wird ersichtlich, dass invektive Adressierungen von Publikum, Medien und Politik zu den zentralen Techniken artivistischer Interventionen gehören. Invektivität ist eine wichtige Ressource der anvisierten Aufstörung des common sense. Zum anderen zeigt sich aber auch, dass bei Schlingensief und dem ZPS ganz unterschiedliche "Politiken der Form" 57 zum Tragen kommen, deren Komplexitätsunterschiede wiederum auf die metareflexive Durchdringung der unterschiedlichen Dimensionen von Herabsetzung durchschlagen. Wie schon Schlingensief mit seiner Container-Aktion, arbeitet auch das ZPS an einer Neuformatierung des öffentlichen Raums durch eine avancierte Verschaltung von Performance, Installation und medialer Proliferation. Hier wie dort gehört die resonanzkalkulierende Arbeit mit einem „invektiven Bildakt"58 zur konzeptionellen Essenz der jeweiligen Aktion. Für die aufmerksamkeitsökonomische Durchschlagskraft braucht es die Dopplung, Verdichtung und Zirkulation der Live-Performance im affektbesetzten "Streit-Bild", in dem "etwa Unerhörtes, Unverständliches, Widersinniges sich Raum schafft." 59 Erst in der medialen Zirkulation der bildlichen Verdichtung, an die sich in den Austauschprozessen des hybriden Mediensystems weitere Stimmen, Wertungen und (Selbst-)Positionierungen anlagern, finden die beiden Aktionen ihre angestrebte Resonanzstärke. Die Ko-Präsenz von Performer*innen und Publikum ist der Kristallisationskern eines disruptiven Geschehens, das in der Vor- und Nachbereitung wie auch in der Live-Berichterstattung immer schon als ein sich entgrenzendes Medienereignis angelegt ist.

Trotz dieser Gemeinsamkeit des Mediengebrauchs, der das ZPS durchaus in eine Traditi-

57 Vgl. Kappelhoff (2010) Utopie Film.

58 Der Terminus verdankt sich einem Debattenvorschlag von Hartmut Böhme, der die zu einseitige Textorientierung der bisherigen Theoriebildung zur Invektivität bemängelt. Im Anschluss an Horst Bredekamps Konzeption ,substitutiver Bildakte' und in Auseinandersetzung mit den bildtheoretischen Arbeiten W.J.T. Mitchells wird es zukünftig in einer Ausdifferenzierung der Begriffsbildung darum gehen müssen, die handlungs- und wirklichkeitsstiftende Wirkung von invektiven Bildern in ihren liminalen, transgressiven Dimensionen genauer zu fassen. Vgl. Bredekamp (2010) Theorie des Bildakts; Mitchell (2008) Bilder verletzen.

59 Sonderegger (2010) Affirmative Kritik, S. 41. 
onslinie Schlingensiefs stellt, sind aber hinsichtlich der narrativen Kontextualisierung, innerhalb derer diese Bild-Strategien ihr Affizierungspotenzial entfalten, deutliche Unterschiede auszumachen. Während Schlingensief vor allem darauf abzielt, ein Krisenexperiment mit offenem Ausgang zu veranstalten, ${ }^{60}$ zeigt sich das ästhetische Dispositiv der ZPS-Aktion von einer eindeutigen moralischen Positionierung organisiert, die die Teilnehmenden gegen Irritationserfahrungen und Deutungsunsicherheiten schützt und "Läuterung und Erlösung" 61 in Aussicht stellt. Hier ist immer schon klar, was richtig und falsch ist und wie zwischen beidem unterschieden werden kann. Ein Gefühl von "unease and discomfort rather then belonging"62, das die Kunsttheoretikern Claire Bishop als produktiven Effekt der Partizipation für die relationale Ästhetik artivistischer Interventionen in Anschlag bringt, stellt sich in den Handlungs- und Begründungsarrangements des ZPS gerade nicht ein. Im Fall von Flüchtlinge fressen wird selbst das schlussendliche Scheitern der Aktion - es kam zu keiner Gesetzesänderung, auch gab es keine Sondererlaubnis zur Überführung von 100 Fluchtwilligen per Charterflugzeug aus Syrien nach Deutschland ${ }^{63}$ - in der Anschlussrede der syrischen Schauspielerin und Dissidentin May Skaf zum symbolischen Triumph nachhaltiger Aufstörung umgedeutet:

Wir waren das falsche Bild in euren Augen, eine Störung. Wir waren für zwei Wochen ein Fehler im System. In einem System, das voller Fehler ist. [...] Wir werden nicht Teil eurer Logik des Tötens sein. [...] Es wäre falsch, im Theater etwas zu Ende zu bringen, was noch lange nicht zu Ende ist. [...] Wir sagen das Finale $a b$, wir ziehen uns zurück. Im Namen der Tiere lassen wir euch mit eurem Dilemma allein. Wir sind nicht die Lösung. Wir sind die traurigen Darsteller eures Untergangs. Der ist zu real, um gespielt zu werden. Die Katharsis findet nicht mehr statt. [...] // Gerne würde ich Ihnen eine große Show bieten, gern

60 Zum Verhältnis von Kunst, Krisenexperiment und Störung vgl. Koch/Nanz (2018) Aesthetic Experiments.

61 Ullrich (2017a) Wahre Meisterwerke, S. 139.

62 Bishop (2004) Antagonism and relational aesthetics, S. 54 .

63 Wohl aber war die Resonanz der Aktion so stark, dass sich der Bundestag auf Initiative der Linken zu einer aktuellen Stunde veranlasst sah, in der über die vom ZPS vorgeschlagenen Handlungsoptionen diskutiert wurde. Vgl. die Zusammenfassung unter https://www.youtube.com/ watch?v=JRbYhNqJoPY (letzter Zugriff 25.04.2020). würde ich vor Ihnen schreien um mein Leben, um Sie alle zu erschüttern. Aber ich kann Ihnen diese Bilder nicht schenken. Was wäre mein Schrei gegen die ungehörten Hilferufe nachts auf dem Meer? Ich verlasse das Theater, ich werde meiner Rolle nicht gerecht. Ich gebe sie an euch ab. An die weiße Bevölkerung Europas. Ihr seid es, die in den Käfig gehen müssen, denn mein Tod hätte in den Augen eurer Politiker kein Gewicht. Ihr müsst übernehmen. Ich bin gescheitert. Das Gesetz ist geblieben. Menschen werden weiter deswegen sterben. Wendet eure Enttäuschung von mir auf jene, die euch vertreten. Ich werde gehen. Ich gehe in diese Stadt und werde eine unter vielen sein. Vergesst mich, vergesst die Tiger. Denkt an euch und daran, was für Menschen ihr sein wollt. Ich wäre gern die Unruhe in euren Herzen. ${ }^{64}$

Nicht nur der sich hier dokumentierende Hang zum melodramatischen Kitsch, der die Rhetorik des ZPS insgesamt kennzeichnet, erscheint problematisch. Schwerer ins Gewicht fällt die Rollenregie, die in paradigmatischer Weise ein wenig reflektiertes, eurozentrisches Sendungsbewusstsein anschaulich werden lässt. Die Rede ist - im Zitat markiert durch den Doppelstrich - zweigeteilt. Während zunächst aus der Fokalisierung der Tiger der finale Akt des, großen Fressens' verweigert wird, richtet im zweiten Teil May Skaf die Stimme an die deutsche Zivilgesellschaft. Beide Teile - und darin setzt sich ein Paternalismus fort, der auch schon die Aktion Die Toten kommen charakterisierte - blenden völlig das asymmetrische Machtgefälle aus, das das eigene politische Verhältnis zu jenen begründet, für die das ZPS zu sprechen und zu agieren behauptet. Auch wenn May Skaf in der Verkündung des finalen Appells ihre Stimme erheben darf, ist es doch eigentlich das privilegierte weiße, mitteleuropäische Künstler*innen-Kollektiv, das von einer scheinbar unfehlbaren moralischen Position aus eine Anklage gegen die deutsche Politik artikuliert und apodiktische Forderungen an die Zivilgesellschaft richtet. Als Nebeneffekt der moralischen Souveränitätsgeste, mit der das ZPS, das Recht des Stärkeren' für sich reklamiert, der sich „für die Rechte des Schwächeren"65 einsetzt, kommt

64 Die Abschlussrede vom 28.06.2016 ist auf der Homepage des Maxim-Gorki-Theaters dokumentiert. Vgl. https://www.gorki.de/de/fluechtlinge-fressen-not-undspiele (letzter Zugriff 28.04.2020).

65 Zitate der ZPS-Homepage unter der Überschrift "Macht und Moral", https://politicalbeauty.de/ueber-das-ZPS. html (letzter Zugriff 20.04.2020). 
es zur Reproduktion der hegemonialen Redeordnung: In deren patriarchal-diskriminierender Logik kann der oder die Andere bloß „Objekt einer Information, niemals [aber] Subjekt in einer Kommunikation"66 sein. Als ob es die Virulenz des Postkolonialismus nicht gäbe, zeigt sich der ,aggressive Humanismus' des ZPS von einem eurozentrischen Sendungsbewusstsein grundiert, das seine Aktionen blind werden lässt für die invektiven Gefahren eines anmaßenden Sprechens, im Namen von'. ${ }^{67}$

Ein weiterer kritischer Punkt, der mit dieser hypermoralischen Aufladung verbunden ist, betrifft die Handlungs- und Haltungsanweisungen, mit denen die ZPS-Aktionen ihr Publikum auf einen gemeinsamen aktivistischen Konsens ausrichten. Folgt man den einschlägigen Aufsätzen von Hauschild und Lewicki68, sind die Aktionen des ZPS ein Paradebeispiel für partizipative Kunst, die aus Zuschauenden activist citizens zu machen in der Lage ist. Angesichts der impliziten Identifikationsoptionen, die das ZPS seinem Publikum zuweist, scheint diese Behauptung jedoch wenig überzeugend. So ist die Möglichkeit der Partizipation durch Design und Paratext der Aktionen immer schon in spezifischer Weise präfiguriert. Zentralinstanz des Geschehens ist und bleibt Philipp Ruch, der die eigene Deutungsmacht nie aufs Spiel setzt und Partizipation nur dann zulässt, wenn die Akzeptanz der Spielregeln außer Frage steht. Bei den ZPS-Aktionen herrscht dementsprechend von vorneherein eine Atmosphäre des Einverständnisses, das den Aktionsraum in einen weitgehend konfliktfreien Raum geteilter Wertschätzung verwandelt. In der von einer "Dialektik des Herzens"69 getriebenen, romantisch-jugendbewegten Moralkommunikation des ZPS exponiert sich - entgegengesetzt zur emphatisch vorgetragenen Rhetorik des Engagements - gerade niemand. An einer Aktion

66 Foucault (1977) Überwachen und Strafen, S. 257. 67 Vgl. hierzu den klassischen Text von Spivak (2007) Can the Subaltern Speak?

68 Hauschild (2016) Von der Kunst Bürger/in zu sein; Lewicki (2017) ,The dead's are coming'.

69 Unter der Formel „Dialektik des Herzens" fasste Helmuth Plessner 1924 die kulturkritische Affektpolitik der Jugendbewegung, die ganz auf das Sozialmodell der Gemeinschaft ausgerichtet war. Irritationsaversion nach innen und moralische Überhöhungsbereitschaft nach außen liefen dabei parallel. Vgl. Plessner (2001) Gemeinschaft, S. 12. des ZPS teilzunehmen bietet - mit den kritischen Worten Slavoj Žižeks gesprochen - die attraktive Möglichkeit, ,authentisch' zu leiden und trotzdem das wohltemperierte Leben entspannt fortzusetzen. ${ }^{70}$ Die im Zuge der ZPS-Aktionen von den Beteiligten aufgeführten Gesten der Subversion haben demnach ganz im Gegenteil zur programmatisch erklärten Wirkungsabsicht wohl "eher einen fiktiven Tauschwert im kulturellen Feld als einen Gebrauchswert im politischen Feld. "71

In der Gegenüberstellung zur von Philip Ruch verkörperten, rigorosen "Bekenntniskultur"72, lässt sich anhand von Michel Foucaults Nachdenken über Parrhesia als Form und Forderung einer Kritik hegemonialer Macht zum Abschluss noch einmal deutlich machen, dass sich Schlingensief in einem zentralen Punkt vom „politische[n] Expres-

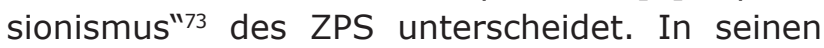
letzten Vorlesungen am Collège de France profiliert Foucault die antike Form der Parrhesia als einen Akt der freimütigen Rede, der Mut erfordert, weil er durch seine rückhaltlose Offenheit riskiert, die Zuneigung des Freundes zu verspielen oder den Zorn des Tyrannen auf sich zu ziehen. Der Parrhesiastes geht ein Risiko dadurch ein, dass er etwas Gefährliches ausspricht - etwas anderes, als das, was sein Gegenüber oder die Mehrheit glaubt. Entscheidend ist dabei, dass er dies tut, ohne die Zustimmung der Gefolgsleute als Rückfalloption zu haben. Im Gegenteil: Die „kynische Parrhesia"74 zeichnet sich dadurch aus, dass der Kritiker, der "ohne Scham und Furcht" und mit "unerträgliche[r] Unverfrorenheit"75 spricht, gerade nicht auf Unterstützung rechnen kann und sich daher selbst aufs Spiel setzt. Anders als in der immunisierenden Souveränitätsgeste Philipp Ruchs, deren moralischer Maximalismus jede differenzierende Nachfrage von vorneherein unter den Generalverdacht der Relativierung stellt, geht es dem Kyniker darum, sich öffentlich zu bestimmten Dingen zu bekennen, auch radikal, um damit eine Situation zu schaffen, „in deren

$\overline{70 ~ V g l}$. Žižek (2000) Substitution zwischen Interaktivität und Interpassivität.

71 Hirsch (2015) Logik der Entscheidung, S. 41.

72 Vgl. Ullrich (2017a) Wahre Meisterwerke, S. 139-143.

73 Ruch (2016) Gespräch mit Ulf Poschardt.

74 Vgl. Wieder (2019) Kritik, Widerstand und das Erbe des Kynismus.

75 Foucault (2010) Regierung des Selbst, S. 218. 
Horizont [...] eine andere Welt"76 als Denkmöglichkeit aufscheint. Entscheidend ist für Foucault die Offenheit des Moments, in dem nicht schon von vorneherein festzulegen ist, wie die Rollen der Zuhörenden, Zuschauenden und Mitwirkenden zu verteilen sind. Diese werden vielmehr erst "im Modus der Konfrontation, des Spotts, der Verhöhnung"77 ausgehandelt. Gewendet auf Schlingensiefs Krisenexperiment bedeutet dies auch, sich selbst, seinen künstlerischen Ansatz und die darin implizierte Tendenz zur Funktionalisierung der im Container bis zu ihrer Abwahl einsitzenden Asylbewerber*innen zur Diskussion zu stellen. Dass hier - wie in jeder Repräsentationspolitik der Aktionskunst - eine kaum zu vermeidende Gefahr der invektiven Bevormundung besteht, macht Bitte liebt Österreich in irritierender Ambiguität dadurch selbst deutlich, dass die im Verlauf der Aktion sonst meist stummen Asylbewerber*innen zusammen mit Elfriede Jelinek ein kleines Puppentheater einstudieren und aufführen. Damit stellt die Aktion aus, unter welchen Bedingungen der Kunstbetrieb die Subalternen sichtbar werden lässt: Als marionettenhafte Objekte ohne eigene Stimme und agency, als bloßes Material einer von privilegierten Weißen gegen die hegemoniale Kultur geäußerten Kritik, die die eigenen performativen Selbstwidersprüche übersieht und sich in der Herrlichkeit einer vermeintlichen moralischen Untadeligkeit sonnt. Wenn man hierfür sensibilisiert auf die Aktionen des ZPS oder auch - um eine andere Lichtgestalt des politisch interessierten Feuilletons zu erwähnen - auf den Theatermacher Milo Rau schaut ${ }^{78}$, so zeigt sich die fast schon aporetische Problematik einer weißen Kritik dominanzkultureller Verhältnisse. ${ }^{79}$ Dort, wo Ruch Partizipation nur

76 Ebd., S. 373.

77 Ebd., S. 360.

78 Im Rahmen der hier angedeuteten Argumentation kann es wenig überraschen, dass Milo Rau und Philipp Ruch derzeit im deutschsprachigen Kontext die mit Abstand resonanzstärksten und populärsten politischen Künstler sind. Beide vereinen eine meinungs- und selbstdeutungsstarke Werkpolitik mit einem Interesse für brisante Themen und dem Willen zur Drastik zu einem ästhetischen Angebot, das in seiner komplexitätsreduzierten moralischen Eindeutigkeit bestens zur Aufmerksamkeitsökonomie und Valorisierungsordnung der sozialen Medien passt.

79 Dass das Verhältnis zu Alterität natürlich auch bei Schlingensief selbst prekär ist, wird insbesondere an seinem Operndorf-Projekt in Burkina Faso deutlich. Vgl. Nier- als Unterordnung unter die eigene moralische Autorität zulässt, besteht der besondere Einsatz der von Schlingensief im Tumult auf dem Wiener Theaterplatz verkörperten Rede in der Bereitschaft, sich selbst, den eigenen Körper und die eigene Position aufs Spiel zu setzen. In der Funktion eines Tricksters handelt er als eine Figur des Dritten, die klare Unterscheidungen unterläuft und „aus Lust an Streichen Widersprüche inszeniert, nicht aus Bosheit, sondern um Leute auf die Probe zu stellen und aus dem Konzept zu bringen." 80 Schlingensief will nicht nur den Anderen auf irritierende Weise die Wahrheit sagen, er ist quasi als ein Neo-Parrhesiastes - auch bereit, die Wahrheit über sich selbst zu sagen, und damit den eigenen Ausschluss aus der Gemeinschaft der kulturräsonierenden Öffentlichkeit wie des politischen Diskurses zu riskieren. ${ }^{81}$

Während das ZPS in der Absetzungsbewegung von der hegemonialen Umgangsweise mit Migration eine eindeutige moralische Haltung im Dienste "moralischer Schönheit, politischer Poesie und menschlicher Großgesinntheit"82 einfordert, setzt Schlingensiefs programmatische Ambiguitätsproduktion analytisch anders an. Die Logik der Störung, die seine Interventionen informiert, zielt nicht allein darauf ab, die Signifikanten gesellschaftlicher Normalitätsproduktion und die damit verbundenen falschen Repräsentationen von Identität und Teilhabeanspruch auszutauschen. Radikaler noch schafft er Situationen,

mann (2013) Operndorf. Er macht sie aber, etwa in seiner Oper Via Intolleranza II, wiederholt zum Thema der eigenen künstlerischen Arbeit.

80 Schüttpelz (2010) Trickster, S. 208.

81 Anders als in Konstellationen des Wahr-Sprechens in der Antike geht es für Schlingensief natürlich nicht um Leben und Tod. Auch muss er nicht riskieren, für seine Intervention ins Gefängnis zu kommen. Wohl aber sind Sanktionen - mediale Stigmatisierung, Verlust von symbolischem Kapital - eine mögliche Konsequenz seiner Aktion. Insofern agiert er, wie mit Judith Butler deutlich zu machen ist, durchaus als Parrhesiastes: „Es gibt andere Arten von Risiken, die nicht mit Strafmaßnahmen der Justiz beziehungsweise der staatlichen Behörden verbunden sind. Man kann sagen, was man für wahr hält, auch wenn es bedeutet, die eigenen Freundinnen und Freunde zu verlieren, sich unbeliebt zu machen oder isoliert zu werden, vielleicht sogar, sich stigmatisiert zu fühlen." Butler (2019) Rücksichtslose Kritik, S. 101.

82 So die programmatische Selbstbeschreibung auf der ZPS-Homepage, https://politicalbeauty.de/ueber-das-ZPS. html (letzter Zugriff 28.04.2020). 
in denen nicht nur Repräsentationen problematisiert, sondern auch die diesen zugrundeliegenden Darstellungsrelationen selbst zum Gegenstand von Beobachtung und Verhandlung werden können. Durch die Produktion von Widersprüchlichkeit, die sich aus der theatralen Suspension der Unterscheidung von dargestellter und tatsächlicher Wirklichkeit ergibt, entsteht erst eine Perspektive, die es erlaubt, die hegemonialen Bedingungen, unter denen Realität bzw. Normalität gesellschaftlich hergestellt wird, einer emanzipierenden Reflexion zugänglich zu machen. ${ }^{83}$ Damit erscheint Schlingensiefs Auseinandersetzung mit der Konstruktion von Fremd- und Andersheit, die viele seiner Interventionen zum Thema haben, als Ausdifferenzierung eines breiteren politischen Projekts: einer Critique of Worldmaking, die zu verstehen versucht, auf Grundlage welcher macht- und invektivgrundierten Bedingungen in der Gesellschaft etwas als etwas erscheint. ${ }^{84}$ Hier erst entsteht die Möglichkeit einer neuen Politisierung, die Einspruch gegen jene herrschenden gouvernementalen Relationalitäten erhebt, auf denen gesellschaftliche Wirklichkeit beruht. Erst in dieser postfundamentalen Wende, so lässt sich mit Nikolaus Müller-Scholl abschließend formulieren, entfaltet das Theater der Diskriminierung, für

83 Womit noch nicht die Frage beantwortet ist, wann und wo diese Reflexion geleistet wird. Kritisch lässt sich zunächst einwenden, dass präsentische Affizierung und distanzierte Reflexion in einem Spannungsfeld zueinanderstehen. Der Vorwurf der Instrumentalisierung der Partizipierenden, der auch im Kontext von Schlingensiefs Arbeit mit Personen mit Behinderungen oft erhoben wurde, ist auch hier nicht völlig von der Hand zu weisen, insofern Schlingensief letztlich aus der ambivalenten Rolle des impulssetzenden Experimentators nicht komplett herauskommt und damit bis zu einem gewissen Grad einen asymmetrischen Wissensvorsprung reproduziert. Mit Jacques Rancière wäre demgegenüber allerdings zu betonen, dass auch das aus der Distanz schauende Interpretieren eine Form der Partizipation ist und Emanzipation demnach das "Verschwimmen der Gegensätze zwischen denen, die betrachten, und denen, die handeln," bedeutet. Rancière (2005b) Spectator, S. 50.

84 Im Sinne Nelson Goodmans: „Worlds are made by making $[. .$.$] versions with words, numerals, pictures, sounds,$ or other symbols of any kind in any medium; and the comparative study of these versions and visions and of their making is what I call a critique of worldmaking." Goodman (1999) Ways of Worldmaking, S. 94. Vgl. hierzu auch Leeten (2012) What is ,Critique of Worldmaking'? das Schlingensief und das ZPS hier exemplarisch herangezogen wurden, sein kritisches Potenzial:

[...] Theater [ist] kritisch nur dort, wo es [...] das Theater aufs Spiel setzt. Wo es sich von jedem Grund, jeder Autorität, jeder Rückversicherung in Regeln, Normen, Institutionen, Konventionen, Handwerk und Technik löst und Spieler*innen und Zuschauer*innen dergestalt im gleichen $M a ß$ an den Rand der eigenen Sicherheit führt wie die philosophische Kritik. Kritisch ist Theater als jene temporäre Überschreitung der Normen, in der diese ausgesetzt und neu verhandelt werden. ${ }^{85}$

\section{Artivismus: Postfundamentalismus vs. Engagement}

Artivistische Interventionen arbeiten an der Produktion von politischen Haltungen, indem sie affektiv hochbesetzte Situationen der Aufstörung schaffen. Sie unterbrechen Normalitätsroutinen und thematisieren das Prekäre einer gesellschaftlichen Wirklichkeit, die sich im Regelbetrieb gerade dadurch stabilisiert, dass sie Fragwürdigkeit in die Latenz verdrängt. Invektivität dient hierbei - dies eint Schlingensief und das ZPS - als symbolische Ressource, um kollektive Wahrnehmungsmuster zu entautomatisieren und eingeübte Kategorialisierungsleistungen zu entsichern. Der zentrale Unterschied zwischen Schlingensief und dem ZPS besteht in der Beantwortung der Frage, wieviel Zutrauen man in die Emanzipationsfähigkeit des Publikums im engeren und der Öffentlichkeit im weiteren Sinne haben kann. Während das ZPS klare Vorstellungen darüber hat, welche gesellschaftlichen Bewusstseinsinhalte falsch und kritikwürdig sind und wodurch sie zu ersetzen wären, verfolgt Schlingensief das Projekt einer Kritik der gesellschaftlichen Modalitäten, unter deren Einfluss sich hegemoniale Bewusstseinsinhalte herausbilden, reproduzieren und aktualisieren. Die Aktionen des ZPS, für die ein "Kippen der Ästhetik in die Ethik" ${ }^{16}$ zu konstatieren ist, betreiben einen großen medialen Aufwand, um auf Missstände hinzuweisen und eine moralische Botschaft an Politik, mediale Öffentlichkeit und Publikum in Szene zu setzen. In gezielter Empörungskommunikation geht es innen um intensive Erlebnisse

85 Müller-Schöll (2018) Fiktion der Kritik, S. 55.

86 Rancière (2016) Unbehagen in der Ästhetik, S. 133. 
der affirmativen Beteiligung. Ihre Aktionen zielen deshalb auf einen konsumierbaren „Happeningeffekt" 87 , der Leute zusammenbringt und innen einen Genuss der gemeinsam erlebten Dissidenz erlaubt. Dafür braucht es Schuldige, die unmissverständlich identifiziert, invektiv adressiert und moralisch verurteilt werden können. ${ }^{88}$ Die implizit anvisierte Homogenität der Wertegemeinschaft hat zur Konsequenz, dass das eigentliche politische Potenzial, das mit einer invektiven Ästhetik der Störung verbunden ist, verloren geht. Ambivalenzen, so Jakob Hayner,

gelten in einer solchen ,Bekenntniskultur' ausschließlich als Störfaktoren. So verkümmert die Form und letztlich auch der Inhalt, denn eine zum Signal degradierte soziale Kritik kann unbekümmert und folgenlos im Modus von like und dislike konsumiert werden [...] Künstlerisches Engagement, das nur auf Bekenntnisse zielt, verfehlt seinen Zweck, schlimmer noch: Es verkehrt diesen in sein Gegenteil. Es befördert eine unkritische Haltung statt einer kritischen. ${ }^{89}$

Schlingensiefs Krisenexperimente dienen demgegenüber zunächst einmal der Ermöglichung von Erfahrungen der Ambiguität und Widersprüchlichkeit. Seine Aktionen sind grell, laut, exzessiv und schonungslos. Allerdings hat bei ihm das Chaos System. Es dient der Herstellung von Inkommensurabilität, was von den Beteiligten als Entsicherung von Konventionen, Destabilisierung von Überzeugungen, verunsichernde Exponierung von Selbst- und Fremdbildern erfahren wird. Gegenüber diesem Programm einer postfundamentalistischen Kritik, die die "Selbstformierung des Subjekts aufs Spiel"90 setzt, wird umso deutlicher, dass das ZPS in seiner Tendenz zur Gesinnungsethik ein Symptom jener post-postmodernen Zeit ist, die durch gesteigerte Moralkommunikation und einen Willen zum Antagonismus geprägt zu sein scheint.

87 Brook (1985) Der leere Raum, S. 94.

88 Diesen Zusammenhang von Selbsterhöhung, Aburteilung und Opfer-Instrumentalisierung führt die Rede von May Skaf ebenso vor wie unlängst die Aktion Sucht nach uns, für bei der die Asche von Holocaust-Opfern in einer Säule vor dem Reichstag als Mahnmal aufgestellt wurde. Vgl. hierzu den Kommentar von Twickel (2019) Diktatur der Anständigen.

89 Hayner (2020) Warum Theater?, S. $83 f$.

90 Butler (2009) Was ist Kritik?, S. 244.

\section{Literaturverzeichnis}

\section{Quellen}

Abschlussrede der Aktion Flüchtlinge fressen vom 28.06.2016, Homepage des Maxim-Gorki-Theaters: https://www.gorki.de/de/fluechtlinge-fressen-notund-spiele (letzter Zugriff 28.04.2020).

DVD Ausländer Raus! Schlingensiefs Container, Bonus Film-GmbH, (Ö 2000), Hoanzl Ö 2002.

Santiago Sierra: $250 \mathrm{~cm}$ Line Tattooed on 6 Paid People Espacio Aglutinador, Havana, Cuba, December 1999, vgl. https://sammlung.staedelmuseum.de/ de/werk/250-cm-line-tattooed-on-6-paid-peopleespacio-aglutinador (letzter Zugriff 20.04.2020).

Selbstbeschreibung auf der Homepage des ZPS: https:// politicalbeauty.de/ (letzter Zugriff 27.04.2020) sowie https://politicalbeauty.de/ueber-das-ZPS.html (letzter Zugriff 28.04.2020).

Zentrum für politische Schönheit: Flüchtlinge fressen. Not und Spiele, Berlin, 2016, vgl. https://politicalbeauty. de/ff.html (letzter Zugriff 27.04.2020).

Zusammenfassung der aktuellen Stunde des Bundestags zur Aktion Flüchtlinge fressen, https://www. youtube.com/watch?v=JRbYhNqJoPY (letzter Zugriff 25.04.2020).

\section{Forschungsliteratur}

Agamben, Giorgio (2002): Homo Sacer. Die souveräne Macht und das nackte Leben. Frankfurt a.M.: Suhrkamp.

Bredekamp, Horst (2010): Theorie des Bildaktes. Frankfurter Adorno-Vorlesungen 2007. Berlin: Suhrkamp.

Bishop, Claire (2004): Antagonism and relational aesthetics. In: October 110, S. 51-79.

Brook, Peter (1985): Der leere Raum. Berlin: Alexander Verlag.

Butler, Judith (2009): Was ist Kritik? Ein Essay über Foucaults Tugend. In: Jaeggi, Rahel/Wesche, Tilo (Hgg.): Was ist Kritik? Frankfurt a.M.: Suhrkamp, S. 221-246.

Butler, Judith (2015): Precariousness and grievability - When is life grievable? In: Verso-Blog, November: https://www.versobooks.com/ blogs/2339-judith-butler-precariousness-andgrievability-when-is-life-grievable (letzter Zugriff 10.02.2019).

Butler, Judith (2019): Rücksichtslose Kritik. Körper, Rede, Aufstand. Konstanz: Konstanz University Press.

Colebrook, Claire (2000): The Meaning of Irony. In: Textual Practices 14/1, S. 5-30.

Diers, Michael (1997): Schlagbilder. Zur politischen Ikonographie der Gegenwart. Frankfurt a.M.: Fischer Taschenbuch.

Foucault, Michel (1977): Überwachen und Strafen. Frankfurt a.M.: Suhrkamp. 
Foucault, Michel (2010): Die Regierung des Selbst und der anderen II. Der Mut zur Wahrheit. Berlin: Suhrkamp.

Gilcher-Holtey, Ingrid (2019): Die Skandalisierung des Skandals. Christoph Schlingensief in der Rolle des Intellektuellen. In: Knapp, Lore/Lindholm, Sven/ Pogoda, Sarah (Hgg.): Christoph Schlingensief und die Avantgarde. Paderborn: Wilhelm Fink, S. 273-292.

Goodman, Nelson (1999): Ways of Worldmaking. Indianapolis: Hackett.

Häusler, Anna/Heyne, Elisabeth/Koch, Lars/Prokic, Tanja (2020): Verletzen und Beleidigen. Versuche zu einer theatralen Kritik der Herabsetzung. Berlin: August.

Hauschild, Alexander (2016): Von der Kunst, Bürger/in zu sein: Politische Imagination, Kreativität und das Zentrum für Politische Schönheit. In: Forschungsjournal Soziale Bewegungen 29/2, S. 50-60.

Hayner, Jakob (2020): Warum Theater? Berlin: Matthes \& Seitz.

Hirsch, Michael (2015): Logik der Entscheidung. Zehn Thesen zu Kunst und Politik. Hamburg: Textem.

Kappelhoff, Hermann (2010): „Ein Denken, das unmittelbar Gefühl, und ein Fühlen, das..." Utopie Film: R. W. Fassbinder und die Frage nach einer „Politik der Form". In: Robnik, Drehli/Hübel, Thomas/ Mattl, Siegfried (Hgg.): Das Streit-Bild. Film, Geschichte und Politik bei Jacques Rancière. Wien/ Berlin: Turia+Kant, S. 161-176.

Kleesattel, Ines (2016): Politische Kunst-Kritik. Zwischen Rancière und Adorno. Wien/Berlin: Turia+Kant.

Koch, Lars (2014): Performance als Bilderstörungsmaschine bei Christoph Schlingensief. In: Zeitschrift für Literaturwissenschaft und Linguistik 173, S. 116-134.

Koch, Lars (2020): Die rechtspopulistische Politik der Gefühle. Angst, Hass, Feindsetzung. In: Ders./ König, Torsten (Hgg.): Zwischen Feindsetzung und Selbstviktimisierung. Gefühlspolitik und Ästhetik populistischer Kommunikation: Frankfurt/New York, S. 87-120.

Koch, Lars/Prokic, Tanja (2022): Die Weißen der Kritik. Über die Aktionen „Die Toten kommen" und "Flüchtlinge fressen. Not und Spiele" des Zentrums für politische Schönheit. In: Böhm, Roswitha/Tiller, Elisabeth (Hgg.): Die mediale Umwelt der Migration. Bielefeld: transcript.

Koch, Lars/Nanz, Tobias (2018): Aesthetic Experiments. On the Event-Like Character and the Function of Disruptions in the Arts. In: Dies./Pause, Johannes (Hgg.): Disruptions in the Arts. Berlin/New York: De Gruyter, S. 3-27.

Koch, Lars/Nanz, Tobias/Pause, Johannes (Hgg.) (2018): Disruptions in the Arts. Textual, Visual, and Performative Strategies for Analyzing Societal Self-Descriptions. Berlin/New York: De Gruyter.

Lehmann, Hans-Thies (2011): Wie politisch ist postdramatisches Theater? In: Deck, Jan/Sieburg, Angelika (Hgg.): Politisch Theater machen. Neue Artikulationsformen des Politischen in den darstellenden Künsten. Bielefeld: transcript, S. 29-40.

Luhmann, Niklas (1996): Die Realität der Massenmedien, Opladen: Westdeutscher Verlag.
Leeten, Lars (2012): What is „Critique of Worldmaking"? Nelson Goodman's conception of philosophy. In: Enrahonar. Quaderns de Filosofia 49, S. 29-40.

Lewicki, Aleksandra (2017): ,The dead are coming': acts of citizenship at Europe's borders. In: Citizenship Studies 21/3, S. 275-290.

Malzacher, Florian (2020): Gesellschaftsspiele. Politisches Theater heute. Berlin: Alexander Verlag.

Marchart, Oliver (2000): Das Licht des Antagonismus. Populärkultur zwischen Mikro-Politik und MakroPolitik. In: Bahlke, Friedrich/Schwering, Gregor/ Stäheli, Urs (Hgg.): Big Brother. Beobachtungen. Bielefeld: transcript, S. 245-260.

Marchart, Oliver (2019): Being agitated - agitated being. Art and activism in times of perennial protest. In: Ders. (Hg.): Conflictual Aesthetics. Artistic Activism and the Public Sphere. Berlin: Sternberg Press, S. 9-50.

Mitchell, William J.T. (2008): Bilder verletzen. In: Ders.: Bildtheorie. Frankfurt a.M.: Suhrkamp, S. 371-395.

Müller-Schöll, Nikolaus (2018): Die Fiktion der Kritik. Foucault, Butler und das Theater der Ent-Unterwerfung. In: Ebert, Olivia et al. (Hgg.): Theater als Kritik. Theorie, Geschichte und Praktiken der Ent-Unterwerfung. Bielefeld: transcript, S. 49-58.

Niermann, Jan Endrik (2013): Schlingensief und das Operndorf Afrika. Analysen der Alterität. Wiesbaden: Springer VS.

o. A. (2000): Aufregung um Wildholz-Sager. In: Wiener Zeitung, 6.06.: https://www.wienerzeitung.at/ nachrichten/politik/oesterreich/350218_Aufregungum-Windholz-Sager.html (letzter Zugriff 23.04.2020).

Pfaller, Robert (2008): Ästhetik der Interpassivität. Hamburg: Philo Fine Arts.

Pinto, Ana Teixeira/Stakemeier, Kerstin (2019): Ein kurzes Glossar zum sozialen Sadismus. In: Texte zur Kunst 116 , S. 69-116.

Plessner, Helmuth (2001): Die Grenzen der Gemeinschaft. Frankfurt a.M.: Suhrkamp.

Pörksen, Bernhard/Schulz von Thun, Friedemann (2020): Die Kunst des Miteinander-Redens. Über den Dialog in Gesellschaft und Politik. München: Carl Hanser.

Rancière, Jacques (2000): Konsens, Dissens, Gewalt. In: Dabag, Mihan/Kapust, Anja/Waldenfels, Bernhard (Hgg.): Gewalt. Strukturen, Formen, Repräsentationen. München: Fink, S. 97-112.

Rancière, Jacques (2002): Das Unvernehmen - Politik und Philosophie. Frankfurt a.M.: Suhrkamp.

Rancière, Jacques (2005a): Die Bestimmung der Bilder. In: Ders.: Politik der Bilder. Berlin: diaphanes, S. 7-42.

Rancière, Jacques (2005b): The emancipated spectator. Ein Vortrag zur Zuschauerperspektive. In: Texte zur Kunst 58, S. 36-52.

Rancière, Jacques (2006): Die Aufteilung des Sinnlichen. Die Politik der Kunst und ihre Paradoxien. Berlin: b-books.

Rancière, Jacques (2008): Zehn Thesen zur Politik. Zürich: diaphanes.

Rancière, Jacques (2011): Moments politiques. Interventionen 1977-2009. Zürich: diaphanes. 
Rancière, Jacques (2016): Das Unbehagen in der Ästhetik. Wien: Passagen Verlag.

Reckwitz, Andreas (2017): Die Gesellschaft der Singularitäten: Zum Strukturwandel der Moderne. Berlin: Suhrkamp.

Ruch, Philipp (2013): Aggressiver Humanismus. Von der Unfähigkeit der Demokratie große Menschenrechtler hervorzubringen. In: Bierdel, Elias/Lakitsch, Maximilian (Hgg.): Wege aus der Krise. Ideen und Konzepte für Morgen. Wien/Münster: LIT, S. 105-119.

Ruch, Philipp (2016): Die Politik ist auf Abschottung konzentriert. Gespräch mit Ulf Poschardt. In: Die Welt, 19.02.: www.welt.de/kultur/article152433895/ Die-Politik-ist-auf-Abschottung-konzentriert.html (letzter Zugriff 10.01.2019).

Scharloth, Joachim (2017): Hassrede und Invektivität als Gegenstand der Sprachwissenschaft und Sprachphilosophie: Bausteine zu einer Theorie des Metainvektiven: In: Aptum 2, S. 116-132.

Schlingensief, Christoph (2014): Ich weiß, ich war's. Köln: Kiepenheuer \& Witsch.

Schüttpelz, Erhard (2010): Der Trickster. In: Eßlinger, Eva et al. (Hgg.): Die Figur des Dritten. Berlin: Suhrkamp, S. 208-224.

Šklovskij, Viktor (1969): Die Kunst als Verfahren. In: Striedter, Jurij (Hg.): Texte der russischen Formalisten. Band I: Texte zur allgemeinen Literaturtheorie und zur Theorie der Prosa. München: Fink, S. 2-35.

Sloterdijk, Peter (2001): Gespräch mit Christoph Schlingensief zur Wienaktion. Homepage Schlingensief: https://www.schlingensief.com/downloads/schlinge_ sloterdijk_wien.pdf (letzter Zugriff 17.08.2020).

Snir, Itay (2016): Not just one common sense: Gramsci's common sense and Laclau and Mouffe's radical democratic politics. In: Constellations 23/2, S. 14-31.

Sonderegger, Ruth (2010): Affirmative Kritik. Wie und warum Jacques Rancière Streit sammelt. In: Robnik, Drehli/Hübel, Thomas/Mattl, Siegfried (Hgg.): Das Streit-Bild. Film, Geschichte und Politik bei Jacques Rancière. Wien: Turia+Kant, S. 29-60.
Spivak, Gayatri Chakravorty (2007): Can the Subaltern Speak? Postkolonialität und subalterne Artikulation. Wien: Turia+Kant.

Stromberg, Matt (2019): Los Angeles gallery opens show with title targeting artist, using ableist slur. In: Hyperallergic, 18.03: https://hyperallergic. com/490175/los-angeles-gallery-opens-show-withtitle-targeting-artist-using-ableist-slur/ (letzter Zugriff 20.04.2020).

Surmann, Frauke (2014): Ästhetische In(ter)ventionen im öffentlichen Raum. Grundzüge einer politischen Ästhetik, Paderborn: Fink.

Twickel, Christoph (2019): Die Diktatur der Anständigen. Das ,Zentrum für Politische Schönheit' tarnt Ideologie als Humanismus. In: Zeit-Online, 17.12.: https:// www.zeit.de/2019/53/zentrum-fuer-politischeschoenheit-aktivismus-humanismus-ideologie (letzter Zugriff 27.04.2020).

Ullrich, Wolfgang (2017a): Wahre Meisterwerte. Stilkritik einer neuen Bekenntniskultur. Berlin: Wagenbach.

Ullrich, Wolfgang (2017b): Die Wiederkehr der Schönheit: Über einige unangenehme Begegnungen. In: Pop-Zeitschrift, 7.11.: https://pop-zeitschrift. de/2017/11/07/die-wiederkehr-der-schoenheit-uebereinige-unangenehme-begegnungenvon-wolfgangullrich07-11-2017/ (letzter Zugriff 17.08.2020).

Wagner, Thomas (2017): Die Angstmacher. 1968 und die Neue Rechte. Berlin: Aufbau.

Warstat, Matthias et al. (2015): Theater als Intervention. Politiken ästhetischer Praxis. Berlin: Theater der Zeit.

Wieder, Anna (2019): Kritik, Widerstand und die Erben des Kynismus. In: Marchart, Oliver/Martinsen, Renate (Hgg.): Foucault und das Politische. Transdisziplinäre Impulse für die politische Theorie der Gegenwart. Wiesbaden: Springer, S. 65-85.

Wihsturz, Benjamin (2014): Der Streit um die Bühne. Theatralität im politischen Denken Jacques Rancières. In: Doll, Martin/Kohns, Oliver (Hgg.): Die imaginäre Dimension der Politik. München: Fink, S. 229-256.

Žižek, Slavoj (2000): Die Substitution zwischen Interaktivität und Interpassivität. In: Pfaller, Robert ( $\mathrm{Hg}$.$) :$ Interpassivität. Studien über delegiertes Genießen. Wien: Springer, S. 13-32. 